\title{
NONLOCAL DELAUNAY SURFACES
}

\author{
JUAN DÁVILA, MANUEL DEL PINO, SERENA DIPIERRO, AND ENRICO VALDINOCI
}

\begin{abstract}
We construct codimension 1 surfaces of any dimension that minimize a periodic nonlocal perimeter functional among surfaces that are periodic, cylindrically symmetric and decreasing.

These surfaces may be seen as a nonlocal analogue of the classical Delaunay surfaces (onduloids). For small volume, most of their mass tends to be concentrated in a periodic array and the surfaces are close to a periodic array of balls (in fact, we give explicit quantitative bounds on these facts).
\end{abstract}

\section{NotATION}

Most of the notation used in this paper is completely standard. For the convenience of the reader, to avoid ambiguities, we state it clearly from the beginning. The standard Euclidean basis of $\mathbb{R}^{n}$ is denoted by $e_{1}, \cdots, e_{n}$ (so that, in particular, $e_{1}=(1,0, \cdots, 0)$ ). If $E \subseteq \mathbb{R}^{n}$ and $v \in \mathbb{R}^{n}$, we use the notation

$$
E+v:=\{p+v, p \in E\} .
$$

Also, $|E|$ is the Lebesgue measure of $E$. The $(n-1)$-dimensional Hausdorff measure is denoted by $\mathscr{H}^{n-1}$. The notation $\Delta$ will be used for the symmetric difference, i.e. $E \Delta F:=(E \backslash F) \cup(F \backslash E)$. We denote by $\chi_{E}$ the characteristic function of a set $E$, i.e.

$$
\chi_{E}(x):= \begin{cases}1 & \text { if } x \in E \\ 0 & \text { if } x \notin E .\end{cases}
$$

Also, throughout the paper, the world "decreasing" stands simply for "non increasing".

\section{INTRODUCTION}

The main goal of this paper is to construct a nonlocal analogue of the classical Delaunay surfaces (see [7]), i.e. surfaces that minimize a fractional perimeter functional among cylindrically decreasing symmetric competitors that are periodic in a given direction. The notion of perimeter that we take into account is a periodic functional of fractional type, whose critical points are related to axially symmetric objects.

We also study the main geometric properties of the minimizers, such as dislocation of mass and closeness to periodic array of balls.

For this scope, we will introduce a new fractional perimeter functional that takes into account the periodicity of the surfaces and we develop a fine analysis of the functional in order to obtain suitable compactness properties. The setting we work in is the following. We consider a fractional parameter $s \in$ $(0,1)$. We use coordinates $x=\left(x_{1}, x^{\prime}\right) \in \mathbb{R} \times \mathbb{R}^{n-1}=\mathbb{R}^{n}$, with $n \geqslant 2$, and deal with the slab

$$
S:=[-1 / 2,1 / 2] \times \mathbb{R}^{n-1} \text {. }
$$

We consider the kernel $K: \mathbb{R}^{n} \backslash\left(\mathbb{Z} \times \mathbb{R}^{n-1}\right) \rightarrow \mathbb{R}$,

$$
K(x):=\sum_{k \in \mathbb{Z}} \frac{1}{\left|x+k e_{1}\right|^{n+s}}
$$

and, given a set $E \subseteq \mathbb{R}^{n}$, we define

$$
P_{S}(E):=\int_{E \cap S} \int_{S \backslash E} K(x-y) d x d y=\int_{E \cap S} \int_{S \backslash E} \sum_{k \in \mathbb{Z}} \frac{d x d y}{\left|x-y+k e_{1}\right|^{n+s}} .
$$

This fractional functional is related to, but quite different from, the nonlocal perimeter introduced in [4] (namely, it shares with it some nonlocal features, but it has different scaling behaviors and periodicity 
properties). More precisely, on the one hand, the functional studied here may be considered as a periodic version (in the horizontal direction) of the fractional perimeter in [4]. On the other hand, the kernel that we consider is non-standard, since it has different scaling properties in the different coordinate directions.

We consider the class of our competitors $\mathscr{K}$, that is given by the sets $F \subseteq S$ of the form

$$
F=\left\{\left(x_{1}, x^{\prime}\right) \in S \text { with }\left|x^{\prime}\right| \leqslant f\left(x_{1}\right)\right\}
$$

for a given even function $f:[-1 / 2,1 / 2] \rightarrow[0,+\infty]$ that is decreasing in $[0,1 / 2]$.

In this setting, we prove the existence of volume constrained minimizers of $P_{S}$ in $\mathscr{K}$ :

Theorem 1. For any $\mu>0$ there exists a minimizer for $P_{S}$ in $\mathscr{K}$ with volume constraint equal to $\mu$.

More explicitly, for any $\mu>0$ there exists a set $F_{*} \in \mathscr{K}$ such that $\left|F_{*}\right|=\mu$ and, for any $F \in \mathscr{K}$ such that $|F|=\mu$, we have that $P_{S}\left(F_{*}\right) \leqslant P_{S}(F)$.

Recently, in the literature, there has been an intense effort towards the construction of geometric object of nonlocal nature that extend classical (i.e. local) ones, see e.g. [6, 9, 8]. In some cases, the nonlocal objects inherit strong geometric properties from the classical case, but also important differences arise. In our setting, we think it is an interesting problem to determine whether cylinders are minimizers for large volume.

As for small volumes, the next result points out (in a quantitative way) that in this case the minimizing set does not put a considerable proportion of mass close to the boundary of the slab (in particular, it is "far from being a cylinder"):

Theorem 2. Let

$$
F_{*}=\left\{\left(x_{1}, x^{\prime}\right) \in S \text { s.t. }\left|x^{\prime}\right| \leqslant f\left(x_{1}\right)\right\}
$$

be a minimizer with volume constraint $\mu>0$, as given in Theorem 1 . Then

$$
\frac{f(1 / 4)}{\mu^{\frac{1}{n-1}}} \leqslant C \mu^{\frac{s}{n^{2}(n-1)}},
$$

for some $C>1$. In particular, for any $\delta \in(0,1)$, if $\mu \in\left(0, C^{-1} \delta^{\frac{n^{2}}{s}}\right)$, we have that

$$
\frac{\left|F_{*} \cap\left\{\left|x_{1}\right| \geqslant 1 / 4\right\}\right|}{\left|F_{*}\right|} \leqslant \delta
$$

for a suitable constant $C>1$.

In case of small volumes, we also show that minimizers are close to balls. The notion of closeness will be measured by the so-called Fraenkel asymmetry (or symmetric deficit) of a set E, which is defined as

$$
\operatorname{Def}(E):=\inf \frac{|E \Delta B|}{|E|},
$$

where the infimum is taken over every ball $B \subset \mathbb{R}^{n}$ with $|B|=|E|$. Roughly speaking, the Fraenkel asymmetry measures the $L^{1}$ distance of $E$ from being a ball of the same volume (the ball may be conveniently translated in order to cover the set $E$ as much as possible, and the quantity above is normalized with respect to the volume in order to be scale invariant). In this setting we have:

Theorem 3. Let $F_{*} \subseteq S$ be a minimizer according to Theorem 1, with volume constraint $\mu$. Then, if $\mu$ is small enough, $F_{*}$ is close to a ball.

More precisely, for any $\mu \in(0,1)$, we have that

$$
\operatorname{Def}\left(F_{*}\right) \leqslant C \mu^{\frac{n^{2}-s^{2}}{2 n^{2}(n-1)}} .
$$

We observe that the control in the sense of deficit obtained in Theorem 3 may lead to further uniform (though perhaps less explicit) asymptotic bounds, also in the $C^{1}$-sense, by exploiting suitable density estimates and approximation results, see e.g. Corollary 3.6 in [9] and Theorem 6.1 in [8].

We point out that, after the present paper was completed and submitted for publication, the very interesting article [3] appeared, dealing with surfaces of constant fractional mean curvature (in the sense 
of [4, 1]). This paper also provides very fascinating fractional counterparts of the Delaunay surfaces (in a different setting than the one considered here, and in the planar case, with an announcement of the $n$-dimensional results to come).

The rest of the paper is organized as follows. In Section 2 we study the decay properties of the kernel. Then, in Section 3 we give a detailed comparison between our functional and the one in [4] (this is not only interesting for seeing similarities and differences with the existing literature, but it is also useful for constructing competitors and deriving estimates).

As a matter of fact, the proof of Theorem 1 also requires a careful energy analysis and ad-hoc compactness arguments in order to use the direct minimization method: these arguments are collected in Sections 4 and 5. With this, all the preliminary work will be completed, and we will be able to prove Theorems 1, 2 and 3 in Sections 6, 7 and 8, respectively.

The paper ends with two appendices. First, in Appendix A, we show that the limit as $s \nearrow 1$ of our fractional functional converges to the classical "periodic" perimeter (i.e. to the perimeter on the cylinder obtained by identifying the "sides" of the slab $S$ ). Then, in Appendix B, we remark that the assumption of cylindrical symmetry for the competitors in $\mathscr{K}$ can be relaxed (in the sense that our fractional functional decreases under cylindrical rearrangements).

\section{KERNEL DECAY}

First, we point out that our functional is compatible with the periodic structure in the horizontal direction. For this, if $F \subseteq S$, we define the periodic extension of $F$ as

$$
F_{\text {per }}:=\bigcup_{k \in \mathbb{Z}}\left(F+k e_{1}\right)
$$

and we have:

Lemma 4. For any $\tau \in \mathbb{R}$, it holds that $P_{S}\left(F_{\text {per }}+\tau e_{1}\right)=P_{S}\left(F_{\text {per }}\right)$.

Proof. The function $x_{1} \mapsto \chi_{F_{\text {per }}}\left(x_{1}, x^{\prime}\right)$ is 1-periodic, and so is $x_{1} \mapsto K\left(x_{1}, x^{\prime}\right)$, for any fixed $x^{\prime} \in \mathbb{R}^{n-1}$. Therefore, for any $\tau \in \mathbb{R}$,

$$
\begin{aligned}
& \int_{[-1 / 2,1 / 2]} d x_{1} \int_{[-1 / 2,1 / 2]} d y_{1} \chi_{F_{\mathrm{per}}+\tau e_{1}}\left(x_{1}, x^{\prime}\right) \chi_{\mathbb{R}^{n} \backslash\left(F_{\mathrm{per}}+\tau e_{1}\right)}\left(y_{1}, y^{\prime}\right) K(x-y) \\
= & \int_{[-1 / 2,1 / 2]-\tau} d x_{1} \int_{[-1 / 2,1 / 2]-\tau} d y_{1} \chi_{F_{\mathrm{per}}}\left(x_{1}, x^{\prime}\right) \chi_{\mathbb{R}^{n} \backslash F_{\mathrm{per}}}\left(y_{1}, y^{\prime}\right) K(x-y) \\
= & \int_{[-1 / 2,1 / 2]} d x_{1} \int_{[-1 / 2,1 / 2]} d y_{1} \chi_{F_{\mathrm{per}}}\left(x_{1}, x^{\prime}\right) \chi_{\mathbb{R}^{n} \backslash F_{\mathrm{per}}}\left(y_{1}, y^{\prime}\right) K(x-y) .
\end{aligned}
$$

Thus the desired result follows by integrating over $x^{\prime}$ and $y^{\prime}$.

Now we prove a useful decay estimate on our kernel. We remark that the scaling properties of our kernel are quite different from the ones of many nonlocal problems that have been studied in the literature: as a matter of fact, the kernel that we study is not homogeneous and it has quite different singular behaviors locally and at infinity. Indeed, close to the origin the dominant term is of the order of $|x|^{-n-s}$, but at infinity the $x_{1}$ direction "averages out", as detailed in the following result:

Lemma 5. For any $x=\left(x_{1}, x^{\prime}\right) \in \mathbb{R}^{n}$ with $\left|x^{\prime}\right| \geqslant 1$, we have that $K(x) \leqslant C\left|x^{\prime}\right|^{1-n-s}$, for some $C>0$.

Proof. Fix $x=\left(x_{1}, x^{\prime}\right) \in \mathbb{R}^{n}$ with $\left|x^{\prime}\right| \geqslant 1$. Let $a_{ \pm}:= \pm\left|x^{\prime}\right|-x_{1}+1$ and $b_{ \pm}:= \pm 3\left|x^{\prime}\right|-x_{1}$. Let also $y_{ \pm}$be the integer part of $b_{ \pm}$. We observe that

$$
b_{+}=3\left|x^{\prime}\right|-x_{1} \geqslant\left|x^{\prime}\right|+2-x_{1}=a_{+}+1 .
$$

This says that at least one integer lies in the segment $\left[a_{+}, b_{+}\right]$and so $y_{+} \geqslant a_{+}$. Therefore

$$
y_{+}+x_{1}-1 \geqslant a_{+}+x_{1}-1=\left|x^{\prime}\right| \text {. }
$$


Moreover

$$
y_{-}+x_{1}+1 \leqslant b_{-}+x_{1}+1 \leqslant-\left|x^{\prime}\right|-1
$$

Consequently

$$
\begin{aligned}
\sum_{\substack{\left.k \in \mathbb{Z} \\
k \in\left(-\infty, b_{-}\right] \cup b_{+},+\infty\right)}} \frac{1}{\left|x_{1}+k\right|^{n+s}} & \leqslant \sum_{\substack{k \in \mathbb{Z} \\
k \geqslant y_{+}}} \frac{1}{\left(x_{1}+k\right)^{n+s}}+\sum_{\substack{k \in \mathbb{Z} \\
k \leqslant y_{-}+1}} \frac{1}{\left(-x_{1}-k\right)^{n+s}} \\
& =\sum_{\substack{k \in \mathbb{Z} \\
k \geqslant y_{+}}} \frac{1}{\left(x_{1}+k\right)^{n+s}}+\sum_{\substack{k \in \mathbb{Z} \\
k \geqslant-y_{-}-1}} \frac{1}{\left(-x_{1}+k\right)^{n+s}} \\
& \leqslant \sum_{\substack{k \in \mathbb{Z} \\
k \geqslant y_{+}}} \int_{x_{1}+k-1}^{x_{1}+k} \frac{d t}{t^{n+s}}+\sum_{\substack{k \in \mathbb{Z} \\
k \geqslant-y_{-}-1}} \int_{-x_{1}+k-1}^{-x_{1}+k} \frac{d t}{t^{n+s}} \\
& =\int_{x_{1}+y_{+}-1}^{+\infty} \frac{d t}{t^{n+s}}+\int_{-x_{1}-y_{-}-2}^{+\infty} \frac{d t}{t^{n+s}} \\
& \leqslant \int_{\left|x^{\prime}\right|}^{+\infty} \frac{d t}{t^{n+s}}+\int_{\left|x^{\prime}\right|}^{+\infty} \frac{d t}{t^{n+s}} \\
& \frac{C}{\left|x^{\prime}\right|^{n+s-1}} .
\end{aligned}
$$

This says that

$$
\sum_{\substack{k \in \mathbb{Z} \\ k \notin\left(-3\left|x^{\prime}\right|-x_{1}, 3\left|x^{\prime}\right|-x_{1}\right)}} \frac{1}{\left|x_{1}+k\right|^{n+s}} \leqslant \frac{C}{\left|x^{\prime}\right|^{n+s-1}} .
$$

Now, we observe that the interval $\left[-3\left|x^{\prime}\right|-x_{1}, 3\left|x^{\prime}\right|-x_{1}\right]$ has length $6\left|x^{\prime}\right|$ and so it contains at most $6\left|x^{\prime}\right|+1 \leqslant$ $7\left|x^{\prime}\right|$ integers. This implies that

$$
\sum_{\substack{k \in \mathbb{Z} \\ k \in\left[-3\left|x^{\prime}\right|-x_{1}, 3\left|x^{\prime}\right|-x_{1}\right]}} \frac{1}{\left|x^{\prime}\right|^{n+s}} \leqslant \frac{7}{\left|x^{\prime}\right|^{n+s-1}} .
$$

Moreover,

$$
\left|x+k e_{1}\right|^{n+s}=\left(\left|x_{1}+k\right|^{2}+\left|x^{\prime}\right|^{2}\right)^{\frac{n+s}{2}} \geqslant \max \left\{\left|x_{1}+k\right|^{n+s},\left|x^{\prime}\right|^{n+s}\right\} .
$$

Thus, recalling (2.1) and (2.2), we conclude that

$$
\begin{aligned}
K(x) & \leqslant \sum_{\substack{k \in \mathbb{Z} \\
k \notin\left(-3\left|x^{\prime}\right|-x_{1}, 3\left|x^{\prime}\right|-x_{1}\right)}} \frac{1}{\left|x+k e_{1}\right|^{n+s}}+\sum_{\substack{k \in \mathbb{Z} \\
k \in\left[-3\left|x^{\prime}\right|-x_{1}, 3\left|x^{\prime}\right|-x_{1}\right]}} \frac{1}{\left|x+k e_{1}\right|^{n+s}} \\
& \leqslant \sum_{\substack{k \in \mathbb{Z} \\
k \notin\left(-3\left|x^{\prime}\right|-x_{1}, 3\left|x^{\prime}\right|-x_{1}\right)}} \frac{1}{\left|x_{1}+k\right|^{n+s}}+\sum_{\substack{k \in \mathbb{Z} \\
k \in\left[-3\left|x^{\prime}\right|-x_{1}, 3\left|x^{\prime}\right|-x_{1}\right]}} \frac{1}{\left|x^{\prime}\right|^{n+s}} \\
& \leqslant \frac{C+7}{\left|x^{\prime}\right|^{n+s-1}},
\end{aligned}
$$

which gives the desired claim up to renaming $C$.

Corollary 6. Fix $M \in \mathbb{N}$. We define

$$
h_{M}(x):=\min \{M, K(x)\} .
$$

Then, for any $x \in \mathbb{R}^{n}$,

$$
h_{M}(x) \leqslant C_{M} \min \left\{1,\left|x^{\prime}\right|^{1-n-s}\right\},
$$

for some $C_{M}>0$ possibly depending on $M$. 
Proof. If $\left|x^{\prime}\right| \geqslant 1$, we use Lemma 5 to see that

$$
\min \left\{1,\left|x^{\prime}\right|^{1-n-s}\right\}=\left|x^{\prime}\right|^{1-n-s} \geqslant C^{-1} K(x) \geqslant C^{-1} h_{M}(x) .
$$

On the other hand, if $\left|x^{\prime}\right|<1$, we have that

$$
\min \left\{1,\left|x^{\prime}\right|^{1-n-s}\right\}=1 \geqslant M^{-1} h_{M}(x) .
$$

Combining these two estimates we obtain the desired result.

\section{Relation With the FRACtional PERimeter}

The aim of this section is to point out the relation between our functional and the fractional perimeter $\mathrm{Per}_{s}$ introduced in [4]. That is, we set

$$
\operatorname{Per}_{s}(F):=\int_{F} \int_{\mathbb{R}^{n} \backslash F} \frac{d x d y}{|x-y|^{n+s}}
$$

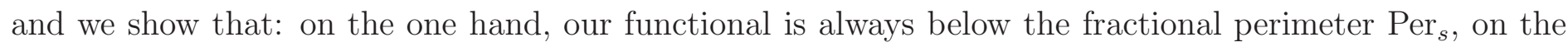
other hand, our functional is always above the fractional perimeter $\mathrm{Per}_{s}$, up to a correction that depends on higher order volume terms, and on a volume term coming from the boundary of the slab $S$. The precise statement goes as follows:

Proposition 7. Let $F \subseteq S$. Then

$$
P_{S}(F) \leqslant \operatorname{Per}_{s}(F)
$$

More precisely,

$$
\operatorname{Per}_{s}(F)-P_{S}(F)=\sum_{k \in \mathbb{Z} \backslash\{0\}} \int_{F} \int_{F} \frac{d x d y}{\left|x-y+k e_{1}\right|^{n+s}}
$$

In addition, if we set

$$
\begin{gathered}
\widetilde{F}:=F \cap\left\{x_{1} \in[1 / 4,1 / 2]\right\}, \\
\widehat{F}:=\left(F+e_{1}\right) \cap\left\{x_{1} \in[1 / 2,3 / 4]\right\} \\
\text { and } \quad \Pi_{S}(F):=\int_{\widetilde{F}} \int_{\widehat{F}} \frac{d x d y}{|x-y|^{n+s}},
\end{gathered}
$$

we have that

$$
\operatorname{Per}_{s}(F) \leqslant P_{S}(F)+C\left(|F|^{2}+\Pi_{S}(F)\right),
$$

for some $C>0$.

Proof. We use the change of variable $\widetilde{y}=y+k e_{1}$ to see that

$$
P_{S}(F)=\int_{F} \int_{S \backslash F} \sum_{k \in \mathbb{Z}} \frac{d x d y}{\left|x-y+k e_{1}\right|^{n+s}}=\int_{F} \int_{(S \backslash F)+k e_{1}} \sum_{k \in \mathbb{Z}} \frac{d x d \widetilde{y}}{|x-\widetilde{y}|^{n+s}}=\int_{F} \int_{(S \backslash F)_{\mathrm{per}}} \frac{d x d \widetilde{y}}{|x-\widetilde{y}|^{n+s}} .
$$

We observe that $(S \backslash F)_{\text {per }} \subseteq \mathbb{R}^{n} \backslash F$, so we obtain that

$$
P_{S}(F) \leqslant \int_{F} \int_{(S \backslash F)_{\mathrm{per}}} \frac{d x d y}{|x-y|^{n+s}} \leqslant \int_{F} \int_{\mathbb{R}^{n} \backslash F} \frac{d x d y}{|x-y|^{n+s}}=\operatorname{Per}_{s}(F) .
$$

This establishes (3.1). More generally, we see that

$$
\left(\mathbb{R}^{n} \backslash F\right) \backslash\left((S \backslash F)_{\text {per }}\right)=\bigcup_{k \in \mathbb{Z} \backslash\{0\}}\left(F+k e_{1}\right),
$$

therefore, with another change of variable, we have

$$
\operatorname{Per}_{s}(F)-P_{S}(F)=\sum_{k \in \mathbb{Z} \backslash\{0\}} \int_{F} \int_{F+k e_{1}} \frac{d x d y}{|x-y|^{n+s}}=\sum_{k \in \mathbb{Z} \backslash\{0\}} \int_{F} \int_{F} \frac{d x d y}{\left|x-y+k e_{1}\right|^{n+s}} \text {. }
$$


This proves (3.2). Now we observe that, if $|k| \geqslant 2$ and $x, y \in S$, then

$$
\left|x-y+k e_{1}\right| \geqslant\left|x_{1}-y_{1}+k\right| \geqslant|k|-\left|x_{1}-y_{1}\right| \geqslant|k|-1 \geqslant \frac{|k|}{2}
$$

therefore

$$
\sum_{\substack{k \in \mathbb{Z} \backslash\{0\} \\|k| \geqslant 2}} \int_{F} \int_{F} \frac{d x d y}{\left|x-y+k e_{1}\right|^{n+s}} \leqslant \sum_{\substack{k \in \mathbb{Z}|\{0\}\\| k \mid \geqslant 2}} \int_{F} \int_{F} \frac{d x d y}{(|k| / 2)^{n+s}} \leqslant C|F|^{2}
$$

for some $C>0$. Moreover,

$$
\int_{F} \int_{F+e_{1}} \chi_{[1 / 4,+\infty)}\left(\left|x_{1}-y_{1}\right|\right) \frac{d x d y}{|x-y|^{n+s}} \leqslant \int_{F} \int_{F+e_{1}} \frac{d x d y}{(1 / 4)^{n+s}} \leqslant C|F|^{2}
$$

for some $C>0$. Also, if $x \in S, y \in S+e_{1}$ and $\left|x_{1}-y_{1}\right| \leqslant 1 / 4$, we have that

$$
\begin{aligned}
& x_{1} \geqslant y_{1}-\left|x_{1}-y_{1}\right| \geqslant-\frac{1}{2}+1-\frac{1}{4}=\frac{1}{4} \\
& \text { and } \quad y_{1}-1 \leqslant\left|y_{1}-x_{1}\right|+x_{1}-1 \leqslant \frac{1}{4}+\frac{1}{2}-1=-\frac{1}{4} .
\end{aligned}
$$

As a consequence, if $x \in F \subseteq S$ and $y_{1} \in F+e_{1} \subseteq S+e_{1}$, with $|x-y| \leqslant 1 / 4$, we have that $x \in \widetilde{F}$ and $y \in \widehat{F}$, where the notation in (3.3) is here in use, therefore

$$
\int_{F} \int_{F+e_{1}} \chi_{[0,1 / 4]}\left(\left|x_{1}-y_{1}\right|\right) \frac{d x d y}{|x-y|^{n+s}} \leqslant \Pi_{S}(F) .
$$

This and (3.7) give that

$$
\int_{F} \int_{F+e_{1}} \frac{d x d y}{|x-y|^{n+s}} \leqslant C\left(|F|^{2}+\Pi_{S}(F)\right) .
$$

Thus, using the change of variable $\bar{x}:=x+e_{1}$ and $\bar{y}:=y+e_{1}$, we also have that

$$
\int_{F} \int_{F-e_{1}} \frac{d x d y}{|x-y|^{n+s}}=\int_{F+e_{1}} \int_{F} \frac{d x d y}{|x-y|^{n+s}} \leqslant C\left(|F|^{2}+\Pi_{S}(F)\right) .
$$

Putting together (3.8) and (3.9) we obtain

$$
\sum_{\substack{k \in \mathbb{Z}\{\{\} \\|k| \leqslant 1}} \int_{F} \int_{F} \frac{d x d y}{\left|x-y+k e_{1}\right|^{n+s}}=\int_{F} \int_{F+e_{1}} \frac{d x d y}{|x-y|^{n+s}}+\int_{F} \int_{F-e_{1}} \frac{d x d y}{|x-y|^{n+s}} \leqslant C\left(|F|^{2}+\Pi_{S}(F)\right) .
$$

This and (3.6) imply that

$$
\sum_{k \in \mathbb{Z} \backslash\{0\}} \int_{F} \int_{F} \frac{d x d y}{\left|x-y+k e_{1}\right|^{n+s}} \leqslant C\left(|F|^{2}+\Pi_{S}(F)\right) .
$$

Recalling (3.5), we see that this ends the proof of (3.4).

As a consequence of our preliminary computations, we obtain that cylinders have finite energy:

Corollary 8. The functional attains a finite value on cylinders. Namely, for any $R>0$, let $\mathscr{C}:=$ $\left\{\left(x_{1}, x^{\prime}\right) \in \mathbb{R} \times \mathbb{R}^{n-1}\right.$ s.t. $\left.\left|x^{\prime}\right| \leqslant R\right\}$. Then $P_{S}(\mathscr{C} \cap S)<+\infty$.

Proof. We take a set $\widetilde{\mathscr{C}}$ with smooth boundary and contained in $[-1,1] \times \mathbb{R}^{n-1}$ such that $\widetilde{\mathscr{C}} \cap S=\mathscr{C} \cap S$. Then, we use (3.1) and we obtain that

$$
+\infty>\operatorname{Per}_{s}(\widetilde{\mathscr{C}}) \geqslant P_{S}(\tilde{\mathscr{C}} \cap S)=P_{S}(\mathscr{C} \cap S),
$$

as desired.

Next result computes the term $\Pi_{S}$ in (3.4) in the special case of small cylinders (this will play a role in the proof of Theorem 3). 
Lemma 9. For any $r \in(0,1 / 4)$, let $\mathscr{C}:=\left\{\left(x_{1}, x^{\prime}\right) \in S\right.$ s.t. $\left.\left|x^{\prime}\right| \leqslant r\right\}$. Then $\Pi_{S}(\mathscr{C}) \leqslant C r^{n-s}$.

Proof. We first translate in the first coordinate and then change variable $X:=x / r$ and $Y:=y / r$, so that we obtain

$$
\begin{aligned}
\Pi_{S}(\mathscr{C}) & =\int_{1 / 4}^{1 / 2} d x_{1} \int_{1 / 2}^{3 / 4} d y_{1} \int_{\left|x^{\prime}\right| \leqslant r} d x^{\prime} \int_{\left|y^{\prime}\right| \leqslant r} d y^{\prime} \frac{1}{|x-y|^{n+s}} \\
& =\int_{-1 / 4}^{0} d x_{1} \int_{0}^{1 / 4} d y_{1} \int_{\left|x^{\prime}\right| \leqslant r} d x^{\prime} \int_{\left|y^{\prime}\right| \leqslant r} d y^{\prime} \frac{1}{|x-y|^{n+s}} \\
& =r^{n-s} \int_{-1 /(4 r)}^{0} d X_{1} \int_{0}^{1 /(4 r)} d Y_{1} \int_{\left|X^{\prime}\right| \leqslant 1} d X^{\prime} \int_{\left|Y^{\prime}\right| \leqslant 1} d Y^{\prime} \frac{1}{|X-Y|^{n+s}}
\end{aligned}
$$

Now we take a bounded set $\mathscr{C}^{\star} \subset[-1,0] \times \mathbb{R}^{n-1}$ with smooth boundary that contains $\left\{\left(x_{1}, x^{\prime}\right) \in\right.$ $\mathbb{R}^{n}$ s.t. $x_{1} \in[-1,0]$ and $\left.\left|x^{\prime}\right| \leqslant 1\right\}$. Then we have that

$$
\int_{-1}^{0} d X_{1} \int_{0}^{1} d Y_{1} \int_{\left|X^{\prime}\right| \leqslant 1} d X^{\prime} \int_{\left|Y^{\prime}\right| \leqslant 1} d Y^{\prime} \frac{1}{|X-Y|^{n+s}} \leqslant \int_{\mathscr{C}^{\star}} \int_{\mathbb{R}^{n} \backslash \mathscr{C}^{\star}} \frac{d X d Y}{|X-Y|^{n+s}} \leqslant \operatorname{Per}_{s}\left(\mathscr{C}^{\star}\right) \leqslant C
$$

for some $C>0$, thus (3.10) becomes

$$
\begin{aligned}
\Pi_{S}(\mathscr{C}) \leqslant C r^{n-s} & \left(1+\int_{-1 /(4 r)}^{-1} d X_{1} \int_{0}^{1 /(4 r)} d Y_{1} \int_{\left|X^{\prime}\right| \leqslant 1} d X^{\prime} \int_{\left|Y^{\prime}\right| \leqslant 1} d Y^{\prime} \frac{1}{|X-Y|^{n+s}}\right. \\
& \left.+\int_{-1 /(4 r)}^{0} d X_{1} \int_{1}^{1 /(4 r)} d Y_{1} \int_{\left|X^{\prime}\right| \leqslant 1} d X^{\prime} \int_{\left|Y^{\prime}\right| \leqslant 1} d Y^{\prime} \frac{1}{|X-Y|^{n+s}}\right) .
\end{aligned}
$$

Notice that we can change variable $(x, y):=(-Y,-X)$ and see that

$$
\begin{array}{r}
\int_{-1 /(4 r)}^{0} d X_{1} \int_{1}^{1 /(4 r)} d Y_{1} \int_{\left|X^{\prime}\right| \leqslant 1} d X^{\prime} \int_{\left|Y^{\prime}\right| \leqslant 1} d Y^{\prime} \frac{1}{|X-Y|^{n+s}} \\
=\int_{0}^{1 /(4 r)} d y_{1} \int_{-1 /(4 r)}^{-1} d x_{1} \int_{\left|y^{\prime}\right| \leqslant 1} d y^{\prime} \int_{\left|x^{\prime}\right| \leqslant 1} d x^{\prime} \frac{1}{|x-y|^{n+s}}
\end{array}
$$

As a consequence, we can write (3.11) as

$$
\Pi_{S}(\mathscr{C}) \leqslant C r^{n-s}\left(1+2 \int_{-1 /(4 r)}^{-1} d x_{1} \int_{0}^{1 /(4 r)} d y_{1} \int_{\left|x^{\prime}\right| \leqslant 1} d x^{\prime} \int_{\left|y^{\prime}\right| \leqslant 1} d y^{\prime} \frac{1}{|x-y|^{n+s}}\right) .
$$

Now we observe that

$$
\begin{aligned}
& \int_{-1 /(4 r)}^{-1} d x_{1} \int_{0}^{1 /(4 r)} d y_{1} \int_{\left|x^{\prime}\right| \leqslant 1} d x^{\prime} \int_{\left|y^{\prime}\right| \leqslant 1} d y^{\prime} \frac{1}{|x-y|^{n+s}} \\
\leqslant & \int_{-1 /(4 r)}^{-1} d x_{1} \int_{0}^{1 /(4 r)} d y_{1} \int_{\left|x^{\prime}\right| \leqslant 1} d x^{\prime} \int_{\left|y^{\prime}\right| \leqslant 1} d y^{\prime} \frac{1}{\left|x_{1}-y_{1}\right|^{n+s}} \\
= & C \int_{-1 /(4 r)}^{-1} d x_{1} \int_{0}^{1 /(4 r)} d y_{1} \frac{1}{\left|x_{1}-y_{1}\right|^{n+s}} \\
\leqslant & C \int_{-1 /(4 r)}^{-1} d x_{1}\left(-x_{1}\right)^{1-n-s} \\
\leqslant & C \int_{1}^{+\infty} d \tau \tau^{1-n-s} \\
\leqslant & C .
\end{aligned}
$$

The desired result thus follows by plugging this estimate into (3.12). 


\section{ENERGY BOUNDS}

We consider here an auxiliary energy functional and we prove that the functional $P_{S}$ is bounded from below by it. The proof requires a very careful analysis of the different contributions and the result, together with the one in the subsequent Proposition 11, will play a crucial role for the proof of Theorem 1, since it will lead to the compactness of the minimizing sequences.

Proposition 10. Let $F \subseteq S$. Suppose that there exists an even function $f:[-1 / 2,1 / 2] \rightarrow[0,+\infty]$, with $f$ decreasing in $[0,1 / 2]$, such that

$$
F:=\left\{\left(x_{1}, x^{\prime}\right) \in S \text { s.t. }\left|x^{\prime}\right| \leqslant f\left(x_{1}\right)\right\} .
$$

Let $\varepsilon_{*} \leqslant \alpha_{*} \in[0,1 / 2]$ and suppose that

$$
f\left(x_{1}\right) \geqslant 4 \text { for all } x_{1} \in\left[0, \varepsilon_{*}\right)
$$

and

$$
f\left(x_{1}\right) \geqslant 2 f\left(y_{1}\right) \text { for all } x_{1} \in\left[0, \varepsilon_{*}\right) \text { and } y_{1} \in\left(\alpha_{*}, 1 / 2\right] \text {. }
$$

Then

$$
P_{S}(F) \geqslant C \int_{0}^{\varepsilon_{*}}\left[\int_{\alpha_{*}}^{1 / 2} \frac{f^{n-1}\left(x_{1}\right)}{\left|x_{1}-y_{1}\right|^{1+s}} d y_{1}\right] d x_{1}
$$

for a suitable constant $C>0$.

Proof. We have

$$
\begin{aligned}
P_{S}(F) & =\int_{F} \int_{S \backslash F} K(x-y) d x d y \\
& \geqslant \int_{F} \int_{S \backslash F} \frac{d x d y}{|x-y|^{n+s}} \\
& =\int_{-1 / 2}^{1 / 2} d x_{1} \int_{-1 / 2}^{1 / 2} d y_{1} \int_{\left\{\left|x^{\prime}\right| \leqslant f\left(x_{1}\right)\right\}} d x^{\prime} \int_{\left\{\left|y^{\prime}\right|>f\left(y_{1}\right)\right\}} d y^{\prime} \frac{1}{|x-y|^{n+s}} .
\end{aligned}
$$

Now we introduce cylindrical coordinates by writing $x^{\prime}=\rho \theta$ and $y^{\prime}=r \omega$, with $\theta, \omega \in S^{n-2}$. We obtain

$$
P_{S}(F) \geqslant \int_{-1 / 2}^{1 / 2} d x_{1} \int_{-1 / 2}^{1 / 2} d y_{1} \int_{S^{n-2}} d \theta \int_{S^{n-2}} d \omega \int_{0}^{f\left(x_{1}\right)} d \rho \int_{f\left(y_{1}\right)}^{+\infty} d r \frac{\rho^{n-2} r^{n-2}}{\left(\left|x_{1}-y_{1}\right|^{2}+|\rho \theta-r \omega|^{2}\right)^{\frac{n+s}{2}}} .
$$

Here and in the sequel, $d \theta$ and $d \omega$ are short notations for $d \mathscr{H}^{n-1}(\theta)$ and $d \mathscr{H}^{n-1}(\omega)$, respectively. Now, for any $\theta \in S^{n-2}$, we consider a rotation $R_{\theta}$ on $S^{n-2}$ such that $\theta=R_{\theta} e_{2}$. Then, we can rotate $\omega=R_{\theta} \widetilde{\omega}$, and obtain that

$$
\begin{array}{rl}
\int_{S^{n-2}} & d \omega \frac{\rho^{n-2} r^{n-2}}{\left(\left|x_{1}-y_{1}\right|^{2}+|\rho \theta-r \omega|^{2}\right)^{\frac{n+s}{2}}}=\int_{S^{n-2}} d \omega \frac{\rho^{n-2} r^{n-2}}{\left(\left|x_{1}-y_{1}\right|^{2}+\left|\rho R_{\theta} e_{2}-r \omega\right|^{2}\right)^{\frac{n+s}{2}}} \\
= & \int_{S^{n-2}} d \widetilde{\omega} \frac{\rho^{n-2} r^{n-2}}{\left(\left|x_{1}-y_{1}\right|^{2}+\left|R_{\theta}\left(\rho e_{2}-r \widetilde{\omega}\right)\right|^{2}\right)^{\frac{n+s}{2}}}=\int_{S^{n-2}} d \widetilde{\omega} \frac{\rho^{n-2} r^{n-2}}{\left(\left|x_{1}-y_{1}\right|^{2}+\left|\rho e_{2}-r \widetilde{\omega}\right|^{2}\right)^{\frac{n+s}{2}}} .
\end{array}
$$

Notice that the latter integral in now independent of $\theta$. Then we can write (4.3) as

$$
P_{S}(F) \geqslant C \int_{-1 / 2}^{1 / 2} d x_{1} \int_{-1 / 2}^{1 / 2} d y_{1} \int_{S^{n-2}} d \omega \int_{0}^{f\left(x_{1}\right)} d \rho \int_{f\left(y_{1}\right)}^{+\infty} d r \frac{\rho^{n-2} r^{n-2}}{\left(\left|x_{1}-y_{1}\right|^{2}+\left|\rho e_{2}-r \omega\right|^{2}\right)^{\frac{n+s}{2}}}
$$

for some $C>0$.

Now we observe that

$$
\left|\rho e_{2}-r \omega\right|^{2}=\rho^{2}+r^{2}-2 \rho r \omega_{2}=|\rho-r|^{2}+2 \rho r\left(1-\omega_{2}\right) .
$$


where $\omega_{2}=\omega \cdot e_{2}$ is the second component of the vector $\omega \in S^{n-2} \subset\{0\} \times \mathbb{R}^{n-1}$. Now 1 we define $\underline{\omega}:=$ $\left(\omega_{3}, \cdots, \omega_{n}\right)$. Then $\omega=\left(0, \omega_{2}, \underline{\omega}\right)$ and

$$
\omega_{2}^{2}+|\underline{\omega}|^{2}=1
$$

We also set

$$
S_{\star}^{n-2}:=\left\{\omega=\left(0, \omega_{2}, \underline{\omega}\right) \in S^{n-2} \text { s.t. } \omega_{2} \geqslant \frac{9}{10}\right\} .
$$

Using (4.5), we see that,

$$
\left\{|\underline{\omega}| \leqslant \frac{\sqrt{19}}{10}\right\} \subseteq S_{\star}^{n-2}
$$

Also, in $S_{\star}^{n-2}$,

$$
2 \rho r\left(1-\omega_{2}\right)=\frac{2 \rho r\left(1-\omega_{2}^{2}\right)}{1+\omega_{2}} \leqslant 2 \rho r\left(1-\omega_{2}^{2}\right)=2 \rho r|\underline{\omega}|^{2} .
$$

Therefore, fixed any $a \geqslant 0$, we have

$$
\begin{aligned}
\int_{S^{n-2}} & \frac{d \omega}{\left(a^{2}+\left|\rho e_{2}-r \omega\right|^{2}\right)^{\frac{n+s}{2}}}=\int_{S^{n-2}} \frac{d \omega}{\left(a^{2}+|\rho-r|^{2}+2 \rho r\left(1-\omega_{2}\right)\right)^{\frac{n+s}{2}}} \\
& \geqslant \int_{S_{\star}^{n-2}} \frac{d \omega}{\left(a^{2}+|\rho-r|^{2}+2 \rho r\left(1-\omega_{2}\right)\right)^{\frac{n+s}{2}}} \geqslant \int_{S_{\star}^{n-2}} \frac{d \omega}{\left(a^{2}+|\rho-r|^{2}+2 \rho r|\underline{\omega}|^{2}\right)^{\frac{n+s}{2}}} \\
& \geqslant C \int_{\{|\underline{\omega}| \leqslant \sqrt{19} / 10\}} \frac{d \underline{\omega}}{\left(a^{2}+|\rho-r|^{2}+2 \rho r|\underline{\omega}|^{2}\right)^{\frac{n+s}{2}}},
\end{aligned}
$$

for some $C>0$, possibly different from line to line. So we use polar coordinates $\mathbb{R}^{n-2} \ni \underline{\omega}=R \varphi$, with $\varphi \in S^{n-3}$ and obtain from the latter estimate that

$$
\int_{S^{n-2}} \frac{d \omega}{\left(a^{2}+\left|\rho e_{2}-r \omega\right|^{2}\right)^{\frac{n+s}{2}}} \geqslant C \int_{0}^{\sqrt{19} / 10} \frac{R^{n-3} d R}{\left(a^{2}+|\rho-r|^{2}+2 \rho r R^{2}\right)^{\frac{n+s}{2}}} .
$$

Now we observe that, for any $X, Y \geqslant 0$, we have that

$$
\int_{0}^{\sqrt{19} / 10} \frac{R^{n-3} d R}{\left(X^{2}+Y^{2} R^{2}\right)^{\frac{n+s}{2}}}=\left(\frac{X}{Y}\right)^{n-2} \frac{1}{X^{n+s}} \int_{0}^{\frac{\sqrt{19} Y}{10 X}} \frac{t^{n-3} d t}{\left(1+t^{2}\right)^{\frac{n+s}{2}}},
$$

where the change of variable $R=X t / Y$ was performed.

Now we denote, for any $x \geqslant 0$,

$$
\Gamma(x):=\int_{0}^{x} \frac{t^{n-3} d t}{\left(1+t^{2}\right)^{\frac{n+s}{2}}} .
$$

We observe that if $x \in[0,1]$ and $t \in[0, x]$, then $1+t^{2} \leqslant 2$ and so

$$
\Gamma(x) \geqslant C \int_{0}^{x} t^{n-3} d t=C x^{n-2}
$$

up to renaming $C>0$. Moreover, if $x \geqslant 1$, then

$$
\Gamma(x) \geqslant \int_{0}^{1} \frac{t^{n-3} d t}{\left(1+t^{2}\right)^{\frac{n+s}{2}}} \geqslant C .
$$

Summarizing, for any $x \geqslant 0$, we have that

$$
\Gamma(x) \geqslant C \min \left\{x^{n-2}, 1\right\} .
$$

\footnotetext{
${ }^{1}$ For concreteness we suppose in this part that $n \geqslant 3$. In the very special case $n=2$, one does not have any component $\left(\omega_{3}, \cdots, \omega_{n}\right)$, so she or he can just disregard $\underline{\omega}$ and go directly to (4.10).
} 
Thus, going back to (4.7),

$$
\begin{aligned}
& \int_{0}^{\sqrt{19} / 10} \frac{R^{n-3} d R}{\left(X^{2}+Y^{2} R^{2}\right)^{\frac{n+s}{2}}}=\left(\frac{X}{Y}\right)^{n-2} \frac{1}{X^{n+s}} \Gamma\left(\frac{\sqrt{19} Y}{10 X}\right) \\
& \geqslant C\left(\frac{X}{Y}\right)^{n-2} \frac{1}{X^{n+s}} \min \left\{1,\left(\frac{Y}{X}\right)^{n-2}\right\}=C \frac{1}{X^{n+s}} \min \left\{\left(\frac{X}{Y}\right)^{n-2}, 1\right\} .
\end{aligned}
$$

Now we take $X:=\sqrt{a^{2}+|\rho-r|^{2}}$ and $Y:=\sqrt{2 \rho r}$ and we plug (4.8) into (4.6). In this way we obtain

$$
\int_{S^{n-2}} \frac{d \omega}{\left(a^{2}+\left|\rho e_{2}-r \omega\right|^{2}\right)^{\frac{n+s}{2}}} \geqslant C \frac{1}{\left(a^{2}+|\rho-r|^{2}\right)^{\frac{n+s}{2}}} \min \left\{\left(\frac{a^{2}+|\rho-r|^{2}}{\rho r}\right)^{\frac{n-2}{2}}, 1\right\} .
$$

Hence we take $a:=\left|x_{1}-y_{1}\right|$ and we insert (4.9) into (4.4), obtaining that

$$
\begin{aligned}
P_{S}(F) \geqslant C & \int_{-1 / 2}^{1 / 2} d x_{1} \int_{-1 / 2}^{1 / 2} d y_{1} \int_{0}^{f\left(x_{1}\right)} d \rho \int_{f\left(y_{1}\right)}^{+\infty} d r \\
& \frac{\rho^{n-2} r^{n-2}}{\left(\left|x_{1}-y_{1}\right|^{2}+|\rho-r|^{2}\right)^{\frac{n+s}{2}}} \min \left\{\left(\frac{\left|x_{1}-y_{1}\right|^{2}+|\rho-r|^{2}}{\rho r}\right)^{\frac{n-2}{2}}, 1\right\} .
\end{aligned}
$$

Now we observe that

$$
\begin{aligned}
& \int_{-1 / 2}^{1 / 2} d x_{1} \int_{-1 / 2}^{1 / 2} d y_{1} \int_{0}^{f\left(x_{1}\right)} d \rho \int_{f\left(y_{1}\right)}^{+\infty} d r \\
& \chi_{\left\{\left|x_{1}-y_{1}\right|^{2}+|\rho-r|^{2} \leqslant \rho r\right\}} \frac{\rho^{n-2} r^{n-2}}{\left(\left|x_{1}-y_{1}\right|^{2}+|\rho-r|^{2}\right)^{\frac{n+s}{2}}} \min \left\{\left(\frac{\left|x_{1}-y_{1}\right|^{2}+|\rho-r|^{2}}{\rho r}\right)^{\frac{n-2}{2}}, 1\right\} \\
= & \int_{-1 / 2}^{1 / 2} d x_{1} \int_{-1 / 2}^{1 / 2} d y_{1} \int_{0}^{f\left(x_{1}\right)} d \rho \int_{f\left(y_{1}\right)}^{+\infty} d r \chi_{\left\{\left|x_{1}-y_{1}\right|^{2}+|\rho-r|^{2} \leqslant \rho r\right\}} \frac{\rho^{\frac{n-2}{2}} r^{\frac{n-2}{2}}}{\left(\left|x_{1}-y_{1}\right|^{2}+|\rho-r|^{2}\right)^{1+\frac{s}{2}}} .
\end{aligned}
$$

This and (4.10) give that

$$
P_{S}(F) \geqslant C \int_{-1 / 2}^{1 / 2} d x_{1} \int_{-1 / 2}^{1 / 2} d y_{1} \int_{0}^{f\left(x_{1}\right)} d \rho \int_{f\left(y_{1}\right)}^{+\infty} d r \chi_{\left\{\left|x_{1}-y_{1}\right|^{2}+|\rho-r|^{2} \leqslant \rho r\right\}} \frac{\rho^{\frac{n-2}{2}} r^{\frac{n-2}{2}}}{\left(\left|x_{1}-y_{1}\right|^{2}+|\rho-r|^{2}\right)^{1+\frac{s}{2}}} .
$$

Accordingly, we perform the change of variable $\rho=\left|x_{1}-y_{1}\right| \alpha$ and $r=\left|x_{1}-y_{1}\right| \beta$, so that (4.11) becomes

$$
\begin{aligned}
P_{S}(F) & \geqslant C \int_{-1 / 2}^{1 / 2} d x_{1} \int_{-1 / 2}^{1 / 2} d y_{1} \int_{0}^{\frac{f\left(x_{1}\right)}{\left|x_{1}-y_{1}\right|}} d \alpha \int_{\frac{f\left(y_{1}\right)}{\left|x_{1}-y_{1}\right|}}^{+\infty} d \beta \chi_{\left\{1+|\alpha-\beta|^{2} \leqslant \alpha \beta\right\}} \frac{\left|x_{1}-y_{1}\right|^{n-2-s} \alpha^{\frac{n-2}{2}} \beta^{\frac{n-2}{2}}}{\left(1+|\alpha-\beta|^{2}\right)^{1+\frac{s}{2}}} \\
& \geqslant C \int_{0}^{\varepsilon_{*}} d x_{1} \int_{\alpha_{*}}^{1 / 2} d y_{1} \int_{\frac{9 f\left(x_{1}\right)}{10\left|x_{1}-y_{1}\right|}}^{\frac{f\left(x_{1}\right)}{\left|x_{1}-y_{1}\right|}} d \alpha \int_{\frac{f\left(y_{1}\right)}{\left|x_{1}-y_{1}\right|}}^{+\infty} d \beta \chi_{\left\{1+|\alpha-\beta|^{2} \leqslant \alpha \beta\right\}} \frac{\left|x_{1}-y_{1}\right|^{n-2-s} \alpha^{\frac{n-2}{2}} \beta^{\frac{n-2}{2}}}{\left(1+|\alpha-\beta|^{2}\right)^{1+\frac{s}{2}}},
\end{aligned}
$$

where $\varepsilon_{*}$ and $\alpha_{*}$ were introduced in (4.1) and (4.2). As a matter of fact, using (4.2), we obtain that, in the domain above,

As a consequence

$$
\frac{f\left(y_{1}\right)}{\left|x_{1}-y_{1}\right|} \leqslant \frac{f\left(x_{1}\right)}{2\left|x_{1}-y_{1}\right|}<\frac{9 f\left(x_{1}\right)}{10\left|x_{1}-y_{1}\right|} \leqslant \alpha
$$

$$
\begin{aligned}
P_{S}(F) & \geqslant C \int_{0}^{\varepsilon_{*}} d x_{1} \int_{\alpha_{*}}^{1 / 2} d y_{1} \int_{\frac{9 f\left(x_{1}\right)}{10\left|x_{1}-y_{1}\right|}}^{\frac{f\left(x_{1}\right)}{\left|x_{1}-y_{1}\right|}} d \alpha \int_{\alpha}^{+\infty} d \beta \chi_{\left\{1+|\alpha-\beta|^{2} \leqslant \alpha \beta\right\}} \frac{\left|x_{1}-y_{1}\right|^{n-2-s} \alpha^{\frac{n-2}{2}} \beta^{\frac{n-2}{2}}}{\left(1+|\alpha-\beta|^{2}\right)^{1+\frac{s}{2}}} \\
& \geqslant C \int_{0}^{\varepsilon_{*}} d x_{1} \int_{\alpha_{*}}^{1 / 2} d y_{1} \int_{\frac{9 f\left(x_{1}\right)}{10\left|x_{1}-y_{1}\right|}}^{\frac{f\left(x_{1}\right)}{\left|x_{1}-y_{1}\right|}} d \alpha \int_{\alpha}^{\alpha+1} d \beta \chi_{\left\{1+|\alpha-\beta|^{2} \leqslant \alpha \beta\right\}} \frac{\left|x_{1}-y_{1}\right|^{n-2-s} \alpha^{\frac{n-2}{2}} \beta^{\frac{n-2}{2}}}{\left(1+|\alpha-\beta|^{2}\right)^{1+\frac{s}{2}}} .
\end{aligned}
$$


Now we observe that in the domain above $0 \leqslant \beta-\alpha \leqslant 1$, therefore, recalling (4.1),

$$
1+|\alpha-\beta|^{2} \leqslant 2 \leqslant \frac{f^{2}\left(x_{1}\right)}{8} \leqslant \frac{f^{2}\left(x_{1}\right)}{8\left|x_{1}-y_{1}\right|^{2}}
$$

and

$$
\alpha \beta \geqslant \alpha^{2} \geqslant \frac{81 f^{2}\left(x_{1}\right)}{100\left|x_{1}-y_{1}\right|^{2}}>\frac{f^{2}\left(x_{1}\right)}{8\left|x_{1}-y_{1}\right|^{2}}
$$

that is

$$
1+|\alpha-\beta|^{2}<\alpha \beta
$$

Accordingly, (4.12) boils down to

$$
P_{S}(F) \geqslant C \int_{0}^{\varepsilon_{*}} d x_{1} \int_{\alpha_{*}}^{1 / 2} d y_{1} \int_{\frac{9 f\left(x_{1}\right)}{10\left|x_{1}-y_{1}\right|}}^{\frac{f\left(x_{1}\right)}{\left|x_{1}-y_{1}\right|}} d \alpha \int_{\alpha}^{\alpha+1} d \beta \frac{\left|x_{1}-y_{1}\right|^{n-2-s} \alpha^{\frac{n-2}{2}} \beta^{\frac{n-2}{2}}}{\left(1+|\alpha-\beta|^{2}\right)^{1+\frac{s}{2}}} .
$$

Hence, using again (4.13),

$$
P_{S}(F) \geqslant C \int_{0}^{\varepsilon_{*}} d x_{1} \int_{\alpha_{*}}^{1 / 2} d y_{1} \int_{\frac{9 f\left(x_{1}\right)}{10\left|x_{1}-y_{1}\right|}}^{\frac{f\left(x_{1}\right)}{\left|x_{1}-y_{1}\right|}} d \alpha \int_{\alpha}^{\alpha+1} d \beta \frac{\left|x_{1}-y_{1}\right|^{n-2-s}}{\left(1+|\alpha-\beta|^{2}\right)^{1+\frac{s}{2}}} \cdot \frac{f^{n-2}\left(x_{1}\right)}{\left|x_{1}-y_{1}\right|^{n-2}} .
$$

Now we point out that

$$
\int_{\alpha}^{\alpha+1} \frac{d \beta}{\left(1+|\alpha-\beta|^{2}\right)^{1+\frac{s}{2}}}=\int_{0}^{1} \frac{d \tau}{\left(1+\tau^{2}\right)^{1+\frac{s}{2}}} \geqslant C
$$

and thus we get that

$$
\begin{aligned}
P_{S}(F) & \geqslant C \int_{0}^{\varepsilon_{*}} d x_{1} \int_{\alpha_{*}}^{1 / 2} d y_{1} \int_{\frac{9 f\left(x_{1}\right)}{10\left|x_{1}-y_{1}\right|}}^{\frac{f\left(x_{1}\right)}{\left|x_{1}-y_{1}\right|}} d \alpha\left|x_{1}-y_{1}\right|^{n-2-s} \cdot \frac{f^{n-2}\left(x_{1}\right)}{\left|x_{1}-y_{1}\right|^{n-2}} \\
& \geqslant C \int_{0}^{\varepsilon_{*}} d x_{1} \int_{\alpha_{*}}^{1 / 2} d y_{1}\left|x_{1}-y_{1}\right|^{n-2-s} \cdot \frac{f^{n-2}\left(x_{1}\right)}{\left|x_{1}-y_{1}\right|^{n-2}} \cdot \frac{f\left(x_{1}\right)}{\left|x_{1}-y_{1}\right|}
\end{aligned}
$$

which completes the proof of Proposition 10.

\section{Convergence issues}

Here we show that uniform energy bounds, as the ones obtained in Proposition 10, joined with volume constraints, imply a suitable compactness.

Proposition 11. Let $C_{1}, C_{2}>0$. Consider a sequence of even functions $f_{k}:[-1 / 2,1 / 2] \rightarrow[0,+\infty]$, with $f_{k}$ decreasing in $[0,1 / 2]$. Assume that for any $\varepsilon_{*, k} \leqslant \alpha_{*, k} \in[0,1 / 2]$ such that

$$
f_{k}\left(x_{1}\right) \geqslant 4 \text { for all } x_{1} \in\left[0, \varepsilon_{*, k}\right)
$$

and

$$
f_{k}\left(x_{1}\right) \geqslant 2 f_{k}\left(y_{1}\right) \text { for all } x_{1} \in\left[0, \varepsilon_{*, k}\right) \text { and } y_{1} \in\left(\alpha_{*, k}, 1 / 2\right]
$$

it holds that

$$
\int_{0}^{\varepsilon_{*, k}}\left[\int_{\alpha_{*, k}}^{1 / 2} \frac{f_{k}^{n-1}\left(x_{1}\right)}{\left|x_{1}-y_{1}\right|^{1+s}} d y_{1}\right] d x_{1} \leqslant C_{1}
$$

Suppose also that

$$
\int_{0}^{1 / 2} f_{k}^{n-1}\left(x_{1}\right) d x_{1}=C_{2} .
$$


Then, there exists a function $f$ such that, up to a subsequence, $f_{k} \rightarrow f$ a.e. in $[-1 / 2,1 / 2]$ as $k \rightarrow+\infty$, and

$$
\int_{0}^{1 / 2} f^{n-1}\left(x_{1}\right) d x_{1}=C_{2} .
$$

Proof. First we point out that, for any $r \in[0,1 / 2]$,

$$
C_{2} \geqslant \int_{0}^{r} f_{k}^{n-1}\left(x_{1}\right) d x_{1} \geqslant r \inf _{[0, r]} f_{k}^{n-1}
$$

that is

$$
f_{k}(r)=\inf _{[0, r]} f_{k} \leqslant\left(\frac{C_{2}}{r}\right)^{\frac{1}{n-1}} .
$$

In particular $f_{k}(1 / 2) \leqslant\left(2 C_{2}\right)^{\frac{1}{n-1}}$. So, for any $M>4+\left(2 C_{2}\right)^{\frac{1}{n-1}}$, we are allowed to define

$$
\varepsilon_{k}(M):=\inf \left\{r \in[0,1 / 2] \text { s.t. } f_{k}(r)<M\right\} .
$$

We claim that

$$
\varepsilon_{k}(M) \leqslant \frac{C_{2}}{M^{n-1}} .
$$

Indeed, if $\varepsilon_{k}(M)=0$ we are done, so we assume $\varepsilon_{k}(M)>0$. Then, for any $k$ and $M$ fixed, for any $j \in \mathbb{N}$, we can take $r_{j} \in\left[\left(1-2^{-j}\right) \varepsilon_{k}(M), \varepsilon_{k}(M)\right]$ such that $f_{k}\left(r_{j}\right) \geqslant M$. Hence, from (5.5), we have that

$$
M \leqslant f_{k}\left(r_{j}\right) \leqslant\left(\frac{C_{2}}{r_{j}}\right)^{\frac{1}{n-1}} .
$$

So we pass $j \rightarrow+\infty$ and we obtain

$$
M \leqslant\left(\frac{C_{2}}{\varepsilon_{k}(M)}\right)^{\frac{1}{n-1}}
$$

that proves (5.7).

Now we define $\alpha_{k}(M):=\varepsilon_{k}(M / 2)$. We point out that, if $x_{1} \in\left[0, \varepsilon_{k}(M)\right)$ then $f_{k}\left(x_{1}\right) \geqslant M$, and therefore $f_{k}\left(x_{1}\right) \geqslant 4$. Also, if $y_{1} \in\left(\alpha_{k}(M), 1 / 2\right]=\left(\varepsilon_{k}(M / 2), 1 / 2\right]$, we have that $f_{k}\left(y_{1}\right) \leqslant M / 2$. Accordingly, if $x_{1} \in\left[0, \varepsilon_{k}(M)\right)$ and $y_{1} \in\left(\alpha_{k}(M), 1 / 2\right]$, we have that $f\left(x_{1}\right) \geqslant M \geqslant 2 f\left(y_{1}\right)$.

These considerations show that (5.1) and (5.2) are satisfied by $\varepsilon_{*, k}:=\varepsilon_{k}(M)$ and $\alpha_{*, k}:=\alpha_{k}(M)$. As a consequence

$$
\text { formula (5.3) holds true with } \varepsilon_{*, k}:=\varepsilon_{k}(M) \text { and } \alpha_{*, k}:=\alpha_{k}(M) \text {. }
$$

Now we claim that there exists a constant $C_{\star}>1$ such that, if $M \geqslant C_{\star}$ then

$$
\int_{0}^{\varepsilon_{k}(M)} f_{k}^{n-1}\left(x_{1}\right) d x_{1} \leqslant \frac{C_{\star}}{M^{s(n-1)}} .
$$

To prove this, we use (5.8) to notice that

$$
C_{1} \geqslant \int_{0}^{\varepsilon_{k}(M)}\left[\int_{\alpha_{k}(M)}^{1 / 2} f_{k}^{n-1}\left(x_{1}\right) \frac{1}{\left|x_{1}-y_{1}\right|^{1+s}} d y_{1}\right] d x_{1} .
$$

Now, fixed $x_{1}<\varepsilon_{k}(M) \leqslant \varepsilon_{k}(M / 2)=\alpha_{k}(M)$, we compute

$$
\int_{\alpha_{k}(M)}^{1 / 2} \frac{1}{\left|x_{1}-y_{1}\right|^{1+s}} d y_{1}=\int_{\alpha_{k}(M)}^{1 / 2} \frac{1}{\left(y_{1}-x_{1}\right)^{1+s}} d y_{1}=\frac{1}{s}\left[\frac{1}{\left(\alpha_{k}(M)-x_{1}\right)^{s}}-\frac{1}{\left((1 / 2)-x_{1}\right)^{s}}\right] .
$$

Now, if $x_{1}<\varepsilon_{k}(M)$, we have that

$$
\frac{1}{2}-x_{1} \geqslant \frac{1}{2}-\varepsilon_{k}(M) \geqslant \frac{1}{2}-\frac{C_{2}}{M^{n-1}} \geqslant \frac{1}{4},
$$


if $M$ is sufficiently large (independently on $k$ ), thanks to (5.7). Moreover, using again (5.7), we see that

$$
\alpha_{k}(M)-x_{1} \leqslant \alpha_{k}(M)=\varepsilon_{k}(M / 2) \leqslant \frac{C_{3}}{M^{n-1}},
$$

for some $C_{3}>0$. Therefore (5.11) implies that

$$
\int_{\alpha_{k}(M)}^{1 / 2} \frac{1}{\left|x_{1}-y_{1}\right|^{1+s}} d y_{1} \geqslant\left[C_{4} M^{s(n-1)}-C_{5}\right] \geqslant C_{6} M^{s(n-1)},
$$

for suitable constants $C_{i}>0$, as long as $M$ is large enough, independently on $k$.

So we plug this information into (5.10), and we conclude that

$$
C_{7} \geqslant M^{s(n-1)} \int_{0}^{\varepsilon_{k}(M)} f_{k}^{n-1}\left(x_{1}\right) d x_{1},
$$

and this proves (5.9) .

Now we claim that

$$
f_{k} \rightarrow f \text { a.e. in }[0,1 / 2] \text {, as } k \rightarrow+\infty,
$$

up to subsequences, for some function $f$. To prove this, we use the compactness of the functions with bounded variation, joined with a diagonal trick. We fix $M \in \mathbb{N}, M \geqslant 1$, and we use (5.7) to see that $\left(\varepsilon_{k}(M), 1 / 2\right) \supseteq\left(C_{2} M^{1-n}, 1 / 2\right)$, for any $k$, and so, by (5.6),

$$
\sup _{\left(C_{2} M^{1-n}, 1 / 2\right)} f_{k} \leqslant \sup _{\left(\varepsilon_{k}(M), 1 / 2\right)} f_{k} \leqslant M .
$$

Since $f_{k}$ is monotone, this gives that $\left\|f_{k}\right\|_{B V\left(C_{2} M^{1-n}, 1 / 2\right)} \leqslant 2 M$. As a consequence of this and of the compactness of bounded variations functions (see e.g. Theorem 3.23 in [2]) we get that, for any fixed $M \in \mathbb{N}$, $f_{k} \rightarrow f^{(M)}$ a.e. in $\left(C_{2} M^{1-n}, 1 / 2\right)$, up to a subsequence, for some function $f^{(M)}:\left(C_{2} M^{1-n}, 1 / 2\right) \rightarrow[0,+\infty]$. More explicitly, we write this subsequence by introducing an increasing function $\phi_{M}: \mathbb{N} \rightarrow \mathbb{N}$, and by stating that

$$
f_{\phi_{M} \mathrm{O} \cdots \circ \phi_{1}(k)} \rightarrow f^{(M)} \text { a.e. in }\left(C_{2} M^{1-n}, 1 / 2\right) .
$$

As a matter of fact, for a.e. $x_{1} \in\left(C_{2} M^{1-n}, 1 / 2\right)$, we have that

$$
f^{(M+1)}\left(x_{1}\right)=\lim _{k \rightarrow+\infty} f_{\phi_{M+1} \circ \phi_{M} \circ \cdots \circ \phi_{1}(k)}\left(x_{1}\right)=\lim _{k \rightarrow+\infty} f_{\phi_{M} \circ \cdots \circ \phi_{1}(k)}\left(x_{1}\right)=f^{(M)}\left(x_{1}\right),
$$

so we can define $f:(0,1 / 2) \rightarrow[0,+\infty]$ by setting $f\left(x_{1}\right):=f^{(M)}\left(x_{1}\right)$, for some $M$ so large that $C_{2} M^{1-n}<$ $x_{1}$. Hence, we consider the diagonal subsequence $f_{\phi_{k} \circ \cdots \circ \phi_{1}(k)}$ and we prove that it converges to $f$ a.e. in $(0,1 / 2)$. For this scope, we fix $\varepsilon>0, x_{1} \in(0,1 / 2)$ (possibly outside a set of measure zero) and $M_{x_{1}} \in \mathbb{N}$ such that $C_{2} M_{x_{1}}^{1-n}<x_{1}$ and we use (5.13) to find $k\left(\varepsilon, x_{1}\right)$ such that, if $k \geqslant k\left(\varepsilon, x_{1}\right)$, then

$$
\left|f_{\phi_{M_{1}} \circ \cdots \circ \phi_{1}(k)}\left(x_{1}\right)-f\left(x_{1}\right)\right| \leqslant \varepsilon \text {. }
$$

Now, if $k \geqslant M_{x_{1}}+k\left(\varepsilon, x_{1}\right)$, we have that $\phi_{k} \circ \cdots \circ \phi_{1}(k)$ is a subsequence of $\phi_{M_{x_{1}}} \circ \cdots \circ \phi_{1}(k)$ and thus

$$
\left|f_{\phi_{k} \circ \cdots \circ \phi_{1}(k)}\left(x_{1}\right)-f\left(x_{1}\right)\right| \leqslant \varepsilon \text {. }
$$

Since $\varepsilon$ is arbitrary, this shows that $f_{\phi_{k} \circ \cdots \circ \phi_{1}(k)}\left(x_{1}\right) \rightarrow f\left(x_{1}\right)$, which in turn completes the proof of (5.12).

Now we identify $f_{k}$ with the subsequence constructed in (5.12) and prove that

$$
f_{k}^{n-1} \rightarrow f^{n-1} \text { in } L^{1}(0,1 / 2), \text { as } k \rightarrow+\infty \text {. }
$$

For this scope, we fix $\delta>0$ and we use (5.12) and Egoroff's Theorem to find $E_{\delta} \subseteq(0,1 / 2)$ such that $\left|(0,1 / 2) \backslash E_{\delta}\right| \leqslant \delta$ and $f_{k}^{n-1} \rightarrow f^{n-1}$ uniformly on $E_{\delta}$. We choose $M_{\delta}:=\delta^{-1 / 2}$ and we use (5.9) to conclude that

$$
\int_{0}^{\varepsilon_{k}\left(M_{\delta}\right)} f_{k}^{n-1}\left(x_{1}\right) d x_{1} \leqslant \frac{C_{\star}}{M_{\delta}^{s(n-1)}}=C_{\star} \delta^{\frac{s(n-1)}{2}},
$$


for every $k$. Therefore

$$
\begin{gathered}
\int_{0}^{1 / 2}\left|f_{k}^{n-1}\left(x_{1}\right)-f_{h}^{n-1}\left(x_{1}\right)\right| d x_{1} \leqslant \int_{(0,1 / 2) \backslash E_{\delta}}\left(f_{k}^{n-1}\left(x_{1}\right)+f_{h}^{n-1}\left(x_{1}\right)\right) d x_{1}+\left\|f_{k}^{n-1}-f_{h}^{n-1}\right\|_{L^{\infty}\left(E_{\delta}\right)}\left|E_{\delta}\right| \\
\leqslant 2 C_{\star} \delta^{\frac{s(n-1)}{2}}+\int_{\left(\varepsilon_{k}\left(M_{\delta}\right), 1 / 2\right) \backslash E_{\delta}} f_{k}^{n-1}\left(x_{1}\right) d x_{1}+\int_{\left(\varepsilon_{h}\left(M_{\delta}\right), 1 / 2\right) \backslash E_{\delta}} f_{h}^{n-1}\left(x_{1}\right) d x_{1}+\left\|f_{k}^{n-1}-f_{h}^{n-1}\right\|_{L^{\infty}\left(E_{\delta}\right)} .
\end{gathered}
$$

Now we recall that, by (5.6),$f_{k} \leqslant M_{\delta}$ in $\left(\varepsilon_{k}\left(M_{\delta}\right), 1 / 2\right)$, thus

$$
\int_{\left(\varepsilon_{k}\left(M_{\delta}\right), 1 / 2\right) \backslash E_{\delta}} f_{k}^{n-1}\left(x_{1}\right) d x_{1} \leqslant M_{\delta}\left|\left(\varepsilon_{k}\left(M_{\delta}\right), 1 / 2\right) \backslash E_{\delta}\right| \leqslant M_{\delta} \delta=\sqrt{\delta} .
$$

The same holds with $h$ instead of $k$. Consequently, formula (5.15) gives that

$$
\int_{0}^{1 / 2}\left|f_{k}^{n-1}\left(x_{1}\right)-f_{h}^{n-1}\left(x_{1}\right)\right| d x_{1} \leqslant 2 C_{\star} \delta^{\frac{s(n-1)}{2}}+2 \sqrt{\delta}+\left\|f_{k}^{n-1}-f_{h}^{n-1}\right\|_{L^{\infty}\left(E_{\delta}\right)} .
$$

So, if we choose $h, k$ so large that $\left\|f_{k}^{n-1}-f_{h}^{n-1}\right\|_{L^{\infty}\left(E_{\delta}\right)} \leqslant \sqrt{\delta}$, we obtain

$$
\int_{0}^{1 / 2}\left|f_{k}^{n-1}\left(x_{1}\right)-f_{h}^{n-1}\left(x_{1}\right)\right| d x_{1} \leqslant 2 C_{\star} \delta^{\frac{s(n-1)}{2}}+3 \sqrt{\delta} .
$$

Since $\delta$ was arbitrarily fixed, we have just shown that $f_{k}$ is a Cauchy sequence on $L^{1}(0,1 / 2)$, which implies (5.14).

The desired claim now follows from (5.14) and an even reflection in $(-1 / 2,0)$.

\section{Proof of Theorem 1}

The proof uses the direct methods of the calculus of variations, combined with the fine estimates of Propositions 10 and 11. That is, we take a minimizing sequence of sets $F_{k} \in \mathscr{K}$ with $\left|F_{k}\right|=\mu$ and

$$
\lim _{k \rightarrow+\infty} P_{S}\left(F_{k}\right)=\inf _{\substack{F \subseteq S \\|F|=\mu}} P_{S}(F)
$$

Since $F_{k} \in \mathscr{K}$, we have that $F_{k}$ has the form

$$
F_{k}=\left\{\left(x_{1}, x^{\prime}\right) \in S \text { s.t. }\left|x^{\prime}\right| \leqslant f_{k}\left(x_{1}\right)\right\}
$$

with $f_{k}:[-1 / 2,1 / 2] \rightarrow[0,+\infty]$ even and decreasing in $[0,1 / 2]$. We remark that, for any $k \in \mathbb{N}$,

$$
\mu=\left|F_{k}\right|=C_{o} \int_{0}^{1 / 2} f_{k}^{n-1}\left(x_{1}\right) d x_{1}
$$

for some dimensional constant $C_{o}>0$. Also, we can fix a set $F_{o}$ with $P_{S}\left(F_{o}\right)<+\infty$ (recall for instance Corollary 8), and we may assume that

$$
P_{S}\left(F_{k}\right) \leqslant P_{S}\left(F_{o}\right) \text { for every } k \in \mathbb{N} .
$$

We want to prove that

$$
\text { up to a subsequence, } f_{k} \rightarrow f \text { a.e. in }[-1 / 2,1 / 2] \text {, with } C_{o} \int_{0}^{1 / 2} f^{n-1}\left(x_{1}\right) d x_{1}=\mu \text {. }
$$

Indeed, if there is a sequence along which

$$
\sup _{k \in \mathbb{N}}\left\|f_{k}\right\|_{L^{\infty}([-1 / 2,1 / 2])}<+\infty
$$

then

$$
\sup _{k \in \mathbb{N}}\left\|f_{k}^{n-1}\right\|_{B V([-1 / 2,1 / 2])}<+\infty
$$


and so, again up to a subsequence, we can pass to the limit in $L^{1}([-1 / 2,1 / 2])$ and a.e. in $[-1 / 2,1 / 2]$, see e.g. Theorem 3.23 in [2], and obtain (6.3). Thus, we can suppose that

$$
\sup _{k \in \mathbb{N}}\left\|f_{k}\right\|_{L^{\infty}([-1 / 2,1 / 2])}=+\infty \text {. }
$$

In this case, we check that the assumptions of Proposition 11 are satisfied. For this, let $\varepsilon_{*, k} \leqslant \alpha_{*, k} \in[0,1 / 2]$ such that

$$
f_{k}\left(x_{1}\right) \geqslant 4 \text { for all } x_{1} \in\left[0, \varepsilon_{*, k}\right)
$$

and

$$
f_{k}\left(x_{1}\right) \geqslant 2 f_{k}\left(y_{1}\right) \text { for all } x_{1} \in\left[0, \varepsilon_{*, k}\right) \text { and } y_{1} \in\left(\alpha_{*, k}, 1 / 2\right] \text {. }
$$

We observe that (6.4) and (6.5) say that (4.1) and (4.2) are satisfied (for all the indices $k$ ), hence we can use Proposition 10 and conclude that, for every $k \in \mathbb{N}$,

$$
P_{S}\left(F_{k}\right) \geqslant C \int_{0}^{\varepsilon_{*, k}}\left[\int_{\alpha_{*, k}}^{1 / 2} \frac{f_{k}^{n-1}\left(x_{1}\right)}{\left|x_{1}-y_{1}\right|^{1+s}} d y_{1}\right] d x_{1},
$$

for a suitable $C>0$. Therefore, for every $k \in \mathbb{N}$,

$$
P_{S}\left(F_{o}\right) \geqslant C \int_{0}^{\varepsilon_{*, k}}\left[\int_{\alpha_{*, k}}^{1 / 2} \frac{f_{k}^{n-1}\left(x_{1}\right)}{\left|x_{1}-y_{1}\right|^{1+s}} d y_{1}\right] d x_{1},
$$

thanks to (6.2), and this gives that condition (5.3) is satisfied in this case. Consequently, (6.3) follows from Proposition [1].

Thus, we define

$$
F_{*}:=\left\{\left(x_{1}, x^{\prime}\right) \in S \text { s.t. }\left|x^{\prime}\right| \leqslant f\left(x_{1}\right)\right\}
$$

and we show that $F_{*}$ is the desired minimizer. First of all, $\left|F_{*}\right|=\mu$, thanks to the integral constraint in (6.3). Furthermore, as $k \rightarrow+\infty$,

$$
\chi_{F_{k}} \rightarrow \chi_{F_{*}} \text { a.e. in } S \text {. }
$$

To check this, we recall that, by (6.3),$f_{k} \rightarrow f$ in $S \backslash Z_{1}$, with $\left|Z_{1}\right|=0$. Moreover, we have that, for any fixed $x_{1} \in[-1 / 2,1 / 2]$, the set $\Lambda^{x_{1}}:=\left\{x^{\prime} \in \mathbb{R}^{n-1}\right.$ s.t. $\left.\left|x^{\prime}\right|=f\left(x_{1}\right)\right\}$ is a sphere in $\mathbb{R}^{n-1}$ and so it is of measure zero in $\mathbb{R}^{n-1}$ (in symbols, $\left|\Lambda^{x_{1}}\right|=0$ ). Thus, if $Z_{2}:=\left\{\left(x_{1}, x^{\prime}\right) \in S\right.$ s.t. $\left.\left|x^{\prime}\right|=f\left(x_{1}\right)\right\}$, we have, by Fubini's Theorem, that

$$
\left|Z_{2}\right|=\int_{-1 / 2}^{1 / 2} d x_{1} \int_{\mathbb{R}^{n-1}} d x^{\prime} \chi_{\left\{f\left(x_{1}\right)=\left|x^{\prime}\right|\right\}}\left(\left|x^{\prime}\right|\right)=\int_{-1 / 2}^{1 / 2} d x_{1}\left|\Lambda^{x_{1}}\right|=0 .
$$

Therefore, (6.6) would follow if we show that $f_{k} \rightarrow f$ in $S \backslash\left(Z_{1} \cup Z_{2}\right)$. For this, fix $x \in S \backslash\left(Z_{1} \cup Z_{2}\right)$. Since $x \notin Z_{2}$ we have that either $\left|x^{\prime}\right|<f\left(x_{1}\right)$ or $\left|x^{\prime}\right|>f\left(x_{1}\right)$. Since $x \notin Z_{1}$ we have that $f_{k}(x) \rightarrow f(x)$, so that for large $k$, either $\left|x^{\prime}\right|<f_{k}\left(x_{1}\right)$ or $\left|x^{\prime}\right|>f_{k}\left(x_{1}\right)$, respectively. This shows that, for large $k, x \in F_{*}$ if and only if $x \in F_{k}$, therefore $\chi_{F_{k}}(x)=\chi_{F}(x)$, and this proves (6.6).

Consequently, using (6.1) and (6.6), we have, by Fatou Lemma,

$$
\begin{aligned}
& \inf _{\substack{F \subseteq S \\
|F|=\mu}} P_{S}(F)=\lim _{k \rightarrow+\infty} P_{S}\left(F_{k}\right)=\lim _{k \rightarrow+\infty} \int_{F_{k}} \int_{S \backslash F_{k}} K(x-y) d x d y \\
& \quad=\lim _{k \rightarrow+\infty} \int_{S} \int_{S} \chi_{F_{k}}(x) \chi_{S \backslash F_{k}}(y) K(x-y) d x d y \geqslant \int_{S} \int_{S} \chi_{F_{*}}(x) \chi_{S \backslash F_{*}}(y) K(x-y) d x d y=P_{S}\left(F_{*}\right) .
\end{aligned}
$$

This shows the desired minimization property and it ends the proof of Theorem [1. 


\section{Proof of Theorem 2}

We suppose that $f(1 / 4) \geqslant \beta \mu^{\frac{1}{n-1}}$, for some $\beta \geqslant 0$, and we obtain an estimate on $\beta$. For this we use the volume constraint and we observe that

$$
C_{o} \mu=\int_{0}^{1} f^{n-1}\left(x_{1}\right) d x_{1} \geqslant \int_{0}^{1 / 8} f^{n-1}\left(x_{1}\right) d x_{1} \geqslant \frac{f^{n-1}(1 / 8)}{8},
$$

for some $C_{o}>0$. That is, by monotonicity, we have that

$$
\beta \mu^{\frac{1}{n-1}} \leqslant f(1 / 4) \leqslant f\left(x_{1}\right) \leqslant f(1 / 8) \leqslant C_{1} \mu^{\frac{1}{n-1}}
$$

for every $x_{1} \in[1 / 8,1 / 4]$ (here and in the sequel $C_{i}>0$ is an appropriate dimensional constant). Notice that this already says that

$$
\beta \leqslant C_{1} .
$$

As a consequence, we see that (1.1) is obvious if $\mu \geqslant 1 / 16^{n-1}$, so we suppose from now on that

$$
\mu \in\left(0,1 / 16^{n-1}\right) .
$$

We let $B$ the ball with volume $\mu$ (say, centered at the origin) and we use the minimality property of $F_{*}$ and (3.1) to see that

$$
\begin{aligned}
C_{2} \mu^{\frac{n-s}{n}} & =\operatorname{Per}_{s}(B) \\
& \geqslant P_{S}(B) \\
& \geqslant P_{S}\left(F_{*}\right) \\
& \geqslant C_{3} \int_{1 / 8}^{1 / 4} d x_{1} \int_{1 / 8}^{1 / 4} d y_{1} \int_{\left|x^{\prime}\right| \leqslant f\left(x_{1}\right)} d x^{\prime} \int_{\left|y^{\prime}\right|>f\left(y_{1}\right)} d y^{\prime} \frac{1}{|x-y|^{n+s}} d \int_{\left|y^{\prime}\right|>C_{1} \mu^{\frac{1}{n-1}}} d y^{\prime} \frac{1}{|x-y|^{n+s}} \\
& \geqslant C_{3} \int_{1 / 8}^{1 / 4} d x_{1} \int_{1 / 8}^{1 / 4} d y_{1} \int_{\left|x^{\prime}\right| \leqslant \beta \mu^{\frac{1}{n-1}}} d x^{\prime} \int
\end{aligned}
$$

Now we let $M:=\mu^{-\frac{1}{n-1}}$. Notice that $M \geqslant 16$, thanks to (17.2), thus, if $N$ is the integer part of $M / 8$, we have that $N \leqslant M / 8$ and

$$
N \geqslant \frac{M}{8}-1 \geqslant \frac{M}{16}
$$

Hence we change variables $X:=M x$ and $Y:=M y$ and then we translate in the first coordinate, and we obtain

$$
\begin{aligned}
C_{4} \mu^{\frac{n-s}{n}} & \geqslant M^{s-n} \int_{M / 8}^{M / 4} d X_{1} \int_{M / 8}^{M / 4} d Y_{1} \int_{\left|X^{\prime}\right| \leqslant \beta M \mu^{\frac{1}{n-1}}} d X^{\prime} \int_{\left|Y^{\prime}\right|>C_{1} M \mu^{\frac{1}{n-1}}} d Y^{\prime} \frac{1}{|X-Y|^{n+s}} \\
& =M^{s-n} \int_{0}^{M / 8} d x_{1} \int_{0}^{M / 8} d y_{1} \int_{\left|x^{\prime}\right| \leqslant \beta} d x^{\prime} \int_{\left|y^{\prime}\right|>C_{1}} d y^{\prime} \frac{1}{|x-y|^{n+s}} \\
& \geqslant M^{s-n} \int_{0}^{N} d x_{1} \int_{0}^{N} d y_{1} \int_{\left|x^{\prime}\right| \leqslant \beta} d x^{\prime} \int_{\left|y^{\prime}\right|>C_{1}} d y^{\prime} \frac{1}{|x-y|^{n+s}} \\
& \geqslant M^{s-n} \sum_{k=1}^{N} \int_{k-1}^{k} d x_{1} \int_{k-1}^{k} d y_{1} \int_{\left|x^{\prime}\right| \leqslant \beta} d x^{\prime} \int_{\left|y^{\prime}\right|>C_{1}} d y^{\prime} \frac{1}{|x-y|^{n+s}} \\
& =M^{s-n} \sum_{k=1}^{N} \int_{0}^{1} d x_{1} \int_{0}^{1} d y_{1} \int_{\left|x^{\prime}\right| \leqslant \beta} d x^{\prime} \int_{\left|y^{\prime}\right|>C_{1}} d y^{\prime} \frac{1}{|x-y|^{n+s}} \\
& =M^{s-n} \int_{0}^{1} d x_{1} d \int_{0}^{1} d \int_{\left|x^{\prime}\right| \leqslant \beta} d x^{\prime} \int_{\left|y^{\prime}\right|>C_{1}} d y^{\prime} \frac{1}{|x-y|^{n+s} .}
\end{aligned}
$$


Now we observe that if $x_{1}, y_{1} \in[0,1],\left|x^{\prime}\right| \leqslant \beta$ and $\left|y^{\prime}\right|>C_{1}+1$, then

$$
\left|x_{1}-y_{1}\right| \leqslant 1 \leqslant C_{1}+1-\beta \leqslant\left|y^{\prime}\right|-\left|x^{\prime}\right| \leqslant\left|x^{\prime}-y^{\prime}\right|,
$$

thanks to (7.1), thus in this case

$$
|x-y| \leqslant C_{5}\left|x^{\prime}-y^{\prime}\right| \leqslant C_{5}\left(\left|x^{\prime}\right|+\left|y^{\prime}\right|\right) \leqslant C_{5}\left(\beta+\left|y^{\prime}\right|\right) \leqslant C_{5}\left(C_{1}+\left|y^{\prime}\right|\right) \leqslant 2 C_{5}\left|y^{\prime}\right| .
$$

Accordingly

$$
\begin{aligned}
& \int_{0}^{1} d x_{1} \int_{0}^{1} d y_{1} \int_{\left|x^{\prime}\right| \leqslant \beta} d x^{\prime} \int_{\left|y^{\prime}\right|>C_{1}} d y^{\prime} \frac{1}{|x-y|^{n+s}} \geqslant \int_{0}^{1} d x_{1} \int_{0}^{1} d y_{1} \int_{\left|x^{\prime}\right| \leqslant \beta} d x^{\prime} \int_{\left|y^{\prime}\right|>C_{1}+1} d y^{\prime} \frac{1}{|x-y|^{n+s}} \\
& \geqslant C_{6} \int_{0}^{1} d x_{1} \int_{0}^{1} d y_{1} \int_{\left|x^{\prime}\right| \leqslant \beta} d x^{\prime} \int_{\left|y^{\prime}\right|>C_{1}+1} d y^{\prime} \frac{1}{\left|y^{\prime}\right|^{n+s}}=C_{7} \beta^{n-1} .
\end{aligned}
$$

By inserting this information into (7.4) and using (7.3) we obtain that

$$
\mu^{\frac{n-s}{n}} \geqslant C_{8} M^{s-n} N \beta^{n-1} \geqslant \frac{C_{8} M^{1+s-n} \beta^{n-1}}{16}=C_{9} \mu^{\frac{n-s-1}{n-1}} \beta^{n-1}
$$

and this gives that $\beta \leqslant C_{10} \mu^{\frac{s}{n^{2}(n-1)}}$. This proves (1.1).

Now we prove (1.2). For this we use the monotonicity of $f$, the volume constraint and (1.1) to compute

$$
\frac{\left|F_{*} \cap\left\{\left|x_{1}\right| \geqslant 1 / 4\right\}\right|}{\left|F_{*}\right|}=C_{11} \mu^{-1} \int_{1 / 4}^{1} f^{n-1}\left(x_{1}\right) d x_{1} \leqslant C_{12} \mu^{-1} f^{n-1}(1 / 4) \leqslant C_{13} \mu^{\frac{s}{n^{2}}},
$$

which implies (1.2). The proof of Theorem 2 is thus complete.

\section{Proof of Theorem 3}

We take $B$ the ball of volume $\mu$ (say, centered at the origin). Using the minimality of $F_{*}$ and Proposition 7 , we see that

$$
0 \leqslant \frac{P_{S}(B)-P_{S}\left(F_{*}\right)}{\mu^{\frac{n-s}{n}}} \leqslant \frac{\operatorname{Per}_{s}(B)-\operatorname{Per}_{s}\left(F_{*}\right)+C\left(\left|F_{*}\right|^{2}+\Pi_{S}\left(F_{*}\right)\right)}{\mu^{\frac{n-s}{n}}} .
$$

Now we use Theorem 2, so we write

$$
F_{*}=\left\{\left(x_{1}, x^{\prime}\right) \in S \text { s.t. }\left|x^{\prime}\right| \leqslant f\left(x_{1}\right)\right\}
$$

with

$$
\frac{f(1 / 4)}{\mu^{\frac{1}{n-1}}} \leqslant C \mu^{\frac{s}{n^{2}(n-1)}}
$$

In particular, using the monotonicity of $f$, we have that, for any $x_{1} \in[1 / 4,1 / 2]$

$$
f\left(x_{1}\right) \leqslant f(1 / 4) \leqslant C \mu^{\frac{s}{n^{2}(n-1)}} \times \mu^{\frac{1}{n-1}}=: r .
$$

This (in the notation of (3.3) ) says that $\widetilde{F}_{*}$ and $\widehat{F}_{*}$ are contained in the cylinder of radius $r$, and therefore, by Lemma 9 ,

$$
\Pi_{S}\left(F_{*}\right) \leqslant C r^{n-s}=C \mu^{\frac{s(n-s)}{n^{2}(n-1)}} \times \mu^{\frac{n-s}{n-1}} .
$$

Using this and the fact that $\left|F_{*}\right|=\mu=|B|$, we write (8.1) as

$$
0 \leqslant \frac{\operatorname{Per}_{s}(B)}{|B|^{\frac{n-s}{n}}}-\frac{\operatorname{Per}_{s}\left(F_{*}\right)}{\left|F_{*}\right|^{\frac{n-s}{n}}}+C\left(\mu^{\frac{n+s}{n}}+\mu^{\frac{n^{2}-s^{2}}{n^{2}(n-1)}}\right)
$$

Now we use the quantitative isoperimetric inequality in Theorem 1.1 of [9], according to which

$$
\frac{\operatorname{Per}_{s}\left(F_{*}\right)}{\left|F_{*}\right|^{\frac{n-s}{n}}} \geqslant \frac{\operatorname{Per}_{s}(B)}{|B|^{\frac{n-s}{n}}}\left(1+C \operatorname{Def}^{2}\left(F^{*}\right)\right) \text {. }
$$


By inserting this into (8.2) we conclude that

$$
0 \leqslant-\frac{\operatorname{Per}_{s}(B)}{|B|^{\frac{n-s}{n}}} C \operatorname{Def}^{2}\left(F^{*}\right)+C\left(\mu^{\frac{n+s}{n}}+\mu^{\frac{n^{2}-s^{2}}{n^{2}(n-1)}}\right)
$$

Since

$$
\frac{n^{2}-s^{2}}{n^{2}(n-1)}=\frac{n-s}{n(n-1)} \frac{(n+s)}{n} \leqslant \frac{n}{n(n-1)} \frac{(n+s)}{n} \leqslant \frac{(n+s)}{n},
$$

we see that (8.3) implies the thesis of Theorem 3 ,

\section{ACKNOWLEDGEMENTS}

J. Dávila and M. del Pino have been supported by grants Fondecyt 1130360 and 1150066, Fondo Basal CMM and by Millenium Nucleus CAPDE NC130017. S. Dipierro has been supported by Alexander von Humboldt-Stiftung. E. Valdinoci has been supported by PRIN grant 201274FYK7 "Critical Point Theory and Perturbative Methods for Nonlinear Differential Equations" and ERC grant 277749 "EPSILON Elliptic Pde's and Symmetry of Interfaces and Layers for Odd Nonlinearities".

\section{Appendix A. Limit of $P_{S}$ AS $s \nearrow 1$}

Now we show that (a suitably scaled version of) our nonlocal perimeter functional $P_{S}$ approaches the classical perimeter as $s \nearrow 1$. Notice that of course the functional $P_{S}$ depends on the fractional parameter $s \in(0,1)$ (though we did not keep track explicitly on this dependence when it was not necessary to use it). Also, heuristically, points "close to each other", up to periodicity, provide the biggest contribution to $P_{S}$, due to the singularity of the kernel. A rigorous version of this concept is given by the following result:

Proposition 12. Let $F \in \mathscr{K}$ be a set with $(\partial F) \cap\left\{\left|x_{1}\right|<1 / 2\right\}$ of class $C^{2}$. Then

$$
\lim _{s \nearrow 1} \frac{1-s}{\omega_{n-1}} P_{S}(F)=\mathscr{H}^{n-1}\left((\partial F) \cap\left\{\left|x_{1}\right|<1 / 2\right\}\right) .
$$

Proof. First of all, we fix $\lambda \in(0,1 / 4)$, to be taken as small as we wish in the sequel, and we define

$$
F_{\lambda}:=F \cap\left\{\left|x_{1}\right| \in\left[\frac{1}{2}-\lambda, \frac{1}{2}\right]\right\} .
$$

We observe that if $x \in F$ and $y \in F \backslash F_{\lambda}$ then

$$
\left|x-y \pm e_{1}\right| \geqslant\left|x_{1}-y_{1} \pm 1\right| \geqslant\left|1 \mp y_{1}\right|-\left|x_{1}\right| \geqslant \frac{1}{2}+\lambda-\left|x_{1}\right| \geqslant \lambda .
$$

Similarly, if $y \in F_{\lambda}$ and $x \in F \backslash F_{\lambda}$ then

$$
\left|x-y \pm e_{1}\right| \geqslant\left|x_{1}-y_{1} \pm 1\right| \geqslant\left|1 \pm x_{1}\right|-\left|y_{1}\right| \geqslant \frac{1}{2}+\lambda-\left|y_{1}\right| \geqslant \lambda .
$$

Consequently,

$$
\sum_{k \in\{-1,+1\}}\left[\int_{F} \int_{F \backslash F_{\lambda}} \frac{d x d y}{\left|x-y+k e_{1}\right|^{n+s}}+\int_{F \backslash F_{\lambda}} \int_{F_{\lambda}} \frac{d x d y}{\left|x-y+k e_{1}\right|^{n+s}}\right] \leqslant 4|F|^{2} \lambda^{-n-s} .
$$

As a consequence

$$
\lim _{s \nearrow 1}(1-s) \sum_{k \in\{-1,+1\}}\left[\int_{F} \int_{F \backslash F_{\lambda}} \frac{d x d y}{\left|x-y+k e_{1}\right|^{n+s}}+\int_{F \backslash F_{\lambda}} \int_{F_{\lambda}} \frac{d x d y}{\left|x-y+k e_{1}\right|^{n+s}}\right]=0,
$$

for any fixed $\lambda \in(0,1 / 4)$.

Now we claim that

$$
\lim _{\lambda \searrow 0} \lim _{s \nearrow 1}(1-s) \sum_{k \in\{-1,+1\}} \int_{F_{\lambda}} \int_{F_{\lambda}} \frac{d x d y}{\left|x-y+k e_{1}\right|^{n+s}}=\omega_{n-1}\left[\mathscr{H}^{n-1}(\partial F)-\mathscr{H}^{n-1}\left((\partial F) \cap\left\{\left|x_{1}\right|<1 / 2\right\}\right)\right] .
$$


To prove this, we write

$$
F_{\lambda}^{+}:=F \cap\left\{x_{1} \in\left[\frac{1}{2}-\lambda, \frac{1}{2}\right]\right\} \text { and } F_{\lambda}^{-}:=F \cap\left\{x_{1} \in\left[-\frac{1}{2},-\frac{1}{2}+\lambda \frac{1}{2}\right]\right\} .
$$

Notice that

$$
F_{\lambda}=F_{\lambda}^{+} \cup F_{\lambda}^{-}
$$

and that if $x \in F_{\lambda}^{+}$and $y \in F_{\lambda}^{+}+e_{1}$, or if $x \in F_{\lambda}^{-}$and $y \in F_{\lambda}^{+}+e_{1}$, or if $x \in F_{\lambda}^{-}$and $y \in F_{\lambda}^{-}+e_{1}$, then we have that $|x-y| \geqslant 1 / 4$, and so

$$
\int_{F_{\lambda}^{+}} \int_{F_{\lambda}^{+}+e_{1}} \frac{d x d y}{|x-y|^{n+s}}+\int_{F_{\lambda}^{-}} \int_{F_{\lambda}^{+}+e_{1}} \frac{d x d y}{|x-y|^{n+s}}+\int_{F_{\lambda}^{-}} \int_{F_{\lambda}^{-}+e_{1}} \frac{d x d y}{|x-y|^{n+s}} \leqslant 3 \cdot 4^{n+s}|F|^{2}
$$

and therefore

$$
\lim _{s \nearrow 1}(1-s)\left[\int_{F_{\lambda}^{+}} \int_{F_{\lambda}^{+}+e_{1}} \frac{d x d y}{|x-y|^{n+s}}+\int_{F_{\lambda}^{-}} \int_{F_{\lambda}^{+}+e_{1}} \frac{d x d y}{|x-y|^{n+s}}+\int_{F_{\lambda}^{-}} \int_{F_{\lambda}^{-}+e_{1}} \frac{d x d y}{|x-y|^{n+s}}\right]=0 .
$$

Now, we define $\Omega_{\lambda}$ as the interior of the closure of $F_{\lambda}^{+} \cup\left(F_{\lambda}^{-}+e_{1}\right)$. By Lemma 11 in [5], we have that

$$
\lim _{s \nearrow 1}(1-s) \int_{F_{\lambda} \cap \Omega_{\lambda}} \int_{\Omega_{\lambda} \backslash F_{\lambda}} \frac{d x d y}{|x-y|^{n+s}}=\omega_{n-1} \operatorname{Per}\left(F_{\lambda}, \Omega_{\lambda}\right)=\omega_{n-1} \mathscr{H}^{n-1}\left(F \cap\left\{x_{1}=1 / 2\right\}\right) .
$$

Accordingly,

$$
\begin{aligned}
& \lim _{\lambda \searrow 0} \lim _{\lambda \nearrow 1}(1-s) \int_{F_{\lambda}^{+}} \int_{F_{\lambda}^{-}+e_{1}} \frac{d x d y}{|x-y|^{n+s}}=\lim _{\lambda \searrow 0 s \nearrow 1} \lim _{\lambda \supset}(1-s) \int_{F_{\lambda} \cap \Omega_{\lambda}} \int_{\Omega_{\lambda} \backslash F_{\lambda}} \frac{d x d y}{|x-y|^{n+s}} \\
& =\omega_{n-1} \mathscr{H}^{n-1}\left(F \cap\left\{x_{1}=1 / 2\right\}\right) .
\end{aligned}
$$

Now, we decompose the set $F_{\lambda}$ as in $(\underline{A .3}$ ) and we change variable, to see that

$$
\begin{aligned}
& \int_{F_{\lambda}} \int_{F_{\lambda}} \frac{d x d y}{\left|x-y-e_{1}\right|^{n+s}}=\int_{F_{\lambda}} \int_{F_{\lambda}+e_{1}} \frac{d x d y}{|x-y|^{n+s}} \\
& \quad=\int_{F_{\lambda}^{-}} \int_{F_{\lambda}^{-}+e_{1}} \frac{d x d y}{|x-y|^{n+s}}+\int_{F_{\lambda}^{-}} \int_{F_{\lambda}^{+}+e_{1}} \frac{d x d y}{|x-y|^{n+s}}+\int_{F_{\lambda}^{+}} \int_{F_{\lambda}^{+}+e_{1}} \frac{d x d y}{|x-y|^{n+s}}+\int_{F_{\lambda}^{+}} \int_{F_{\lambda}^{-}+e_{1}} \frac{d x d y}{|x-y|^{n+s}} .
\end{aligned}
$$

Using this, (A.4) and (A.5), we obtain that

$$
\lim _{\lambda \searrow 0} \lim _{s \nearrow 1}(1-s) \int_{F_{\lambda}} \int_{F_{\lambda}} \frac{d x d y}{\left|x-y-e_{1}\right|^{n+s}}=\omega_{n-1} \mathscr{H}^{n-1}\left(F \cap\left\{x_{1}=1 / 2\right\}\right) .
$$

Similarly, one sees that

$$
\lim _{\lambda \searrow 0} \lim _{s \nearrow 1}(1-s) \int_{F_{\lambda}} \int_{F_{\lambda}} \frac{d x d y}{\left|x-y+e_{1}\right|^{n+s}}=\omega_{n-1} \mathscr{H}^{n-1}\left(F \cap\left\{x_{1}=-1 / 2\right\}\right) .
$$

Notice also that

$$
\mathscr{H}^{n-1}\left(F \cap\left\{x_{1}=-1 / 2\right\}\right)+\mathscr{H}^{n-1}\left(F \cap\left\{x_{1}=1 / 2\right\}\right)+\mathscr{H}^{n-1}\left((\partial F) \cap\left\{\left|x_{1}\right|<1 / 2\right\}\right)=\mathscr{H}^{n-1}(\partial F) .
$$

This, (A.6) and (A.7) give the proof of (A.2).

Now we observe that

$$
\begin{aligned}
& \sum_{k \in\{-1,+1\}} \int_{F} \int_{F} \frac{d x d y}{\left|x-y+k e_{1}\right|^{n+s}} \\
= & \sum_{k \in\{-1,+1\}}\left[\int_{F_{\lambda}} \int_{F_{\lambda}} \frac{d x d y}{\left|x-y+k e_{1}\right|^{n+s}}+\int_{F} \int_{F \backslash F_{\lambda}} \frac{d x d y}{\left|x-y+k e_{1}\right|^{n+s}}+\int_{F \backslash F_{\lambda}} \int_{F_{\lambda}} \frac{d x d y}{\left|x-y+k e_{1}\right|^{n+s}}\right] .
\end{aligned}
$$


Thus, we exploit A.1 and (A.2) and we obtain that

$$
\lim _{s \nearrow 1}(1-s) \sum_{k \in\{-1,+1\}} \int_{F} \int_{F} \frac{d x d y}{\left|x-y+k e_{1}\right|^{n+s}}=\omega_{n-1}\left[\mathscr{H}^{n-1}(\partial F)-\mathscr{H}^{n-1}\left((\partial F) \cap\left\{\left|x_{1}\right|<1 / 2\right\}\right)\right] .
$$

Now we observe that if $|k| \geqslant 2$ and $x, y \in S$, then

$$
\left|x-y+k e_{1}\right| \geqslant|k|-|x-y| \geqslant|k|-1 \geqslant \frac{k}{2},
$$

therefore, for any $s \in[1 / 2,1)$,

$$
\sum_{k \in \mathbb{Z} \backslash\{0,-1,+1\}} \int_{F} \int_{F} \frac{d x d y}{\left|x-y+k e_{1}\right|^{n+s}} \leqslant \sum_{k \in \mathbb{Z} \backslash\{0,-1,+1\}} \frac{2^{n+s}|F|^{2}}{|k|^{n+s}} \leqslant \sum_{k \in \mathbb{Z} \backslash\{0,-1,+1\}} \frac{2^{n+1}|F|^{2}}{|k|^{n+(1 / 2)}},
$$

which implies that

$$
\lim _{s \nearrow 1}(1-s) \sum_{k \in \mathbb{Z} \backslash\{0,-1,+1\}} \int_{F} \int_{F} \frac{d x d y}{\left|x-y+k e_{1}\right|^{n+s}}=0
$$

This and (A.8) yield that

$$
\lim _{s \nearrow 1}(1-s) \sum_{k \in \mathbb{Z} \backslash\{0\}} \int_{F} \int_{F} \frac{d x d y}{\left|x-y+k e_{1}\right|^{n+s}}=\omega_{n-1}\left[\mathscr{H}^{n-1}(\partial F)-\mathscr{H}^{n-1}\left((\partial F) \cap\left\{\left|x_{1}\right|<1 / 2\right\}\right)\right] .
$$

Moreover, by Theorem 1 in [5], we have that

$$
\lim _{s \nearrow 1}(1-s) \operatorname{Per}_{s}(F)=\omega_{n-1} \operatorname{Per}(F)=\omega_{n-1} \mathscr{H}^{n-1}(\partial F)
$$

Using this, (3.2) and (A.9), we conclude that

$$
\begin{aligned}
& \omega_{n-1} \mathscr{H}^{n-1}(\partial F)-\lim _{s \nearrow 1}(1-s) P_{S}(F)=\lim _{s \nearrow 1}(1-s)\left[\operatorname{Per}_{s}(F)-P_{S}(F)\right] \\
& =\lim _{s \nearrow 1}(1-s) \sum_{k \in \mathbb{Z} \backslash\{0\}} \int_{F} \int_{F} \frac{d x d y}{\left|x-y+k e_{1}\right|^{n+s}} \\
& =\omega_{n-1}\left[\mathscr{H}^{n-1}(\partial F)-\mathscr{H}^{n-1}\left((\partial F) \cap\left\{\left|x_{1}\right|<1 / 2\right\}\right)\right],
\end{aligned}
$$

which gives the desired result.

\section{Appendix B. Symmetric Rearrangements in $x^{\prime}$}

Here we show that spherical rearrangements in the variable $x^{\prime} \in \mathbb{R}^{n-1}$ make our functional decrease. Given a set $A \subseteq \mathbb{R}^{n-1}$ (respectively, a function $f: \mathbb{R}^{n-1} \rightarrow[0,+\infty]$ ), we consider its radially symmetricdecreasing rearrangement $A^{*}$ (respectively $f^{*}$, see e.g. pages 80-81 in [10] for basic definition and properties). Given $A \subseteq \mathbb{R}^{n}$ (respectively, $f: \mathbb{R}^{n} \rightarrow \mathbb{R}$ ), fixed any $x_{1} \in \mathbb{R}$ we denote by $A^{x_{1} \text {,* }}$ (respectively, $\left.f^{x_{1}, *}: \mathbb{R}^{n-1} \rightarrow \mathbb{R}\right)$ the radially symmetric-decreasing rearrangement of the set

$$
A^{x_{1}}:=\left\{x^{\prime} \in \mathbb{R}^{n-1} \text { s.t. }\left(x_{1}, x^{\prime}\right) \in A\right\}
$$

(respectively, of the function $f\left(x_{1}, \cdot\right)$ ).

Given $A \subseteq S$ we also set

$$
A^{\star}:=\bigcup_{x_{1} \in[-1 / 2,1 / 2]}\left\{x_{1}\right\} \times A^{x_{1}, *} .
$$

We now show that $P_{S}$ decreases under this radially symmetric-decreasing rearrangement in the variable $x^{\prime}$ :

Proposition 13. For any $F \subseteq S$ with $|F|<+\infty$, we have that $P_{S}\left(F^{\star}\right) \leqslant P_{S}(F)$. 
Proof. Fix $M \in \mathbb{N}$ (to be taken arbitrarily large in what follows). We take $h_{M}$ as in (2.3). Notice that, by Corollary 6 (and up to renaming $C_{M}$ ),

$$
\kappa_{M}:=\int_{S} h_{M}(x) d x \leqslant C_{M} \int_{\mathbb{R}^{n-1}} \min \left\{1,\left|x^{\prime}\right|^{1-n-s}\right\} d x^{\prime} \leqslant C_{M}<+\infty .
$$

Furthermore, $K\left(x_{1}+1, x^{\prime}\right)=K\left(x_{1}, x^{\prime}\right)$. This implies that also the map $x_{1} \mapsto h_{M}\left(x_{1}, x^{\prime}\right)$ is 1 -periodic for any fixed $x^{\prime} \in \mathbb{R}^{n-1}$. Thus we can consider its integral on a period, and we have that, for any $r \in \mathbb{R}$,

$$
\int_{r+[-1 / 2,1 / 2]} h_{M}\left(x_{1}, x^{\prime}\right) d x_{1}=\int_{[-1 / 2,1 / 2]} h_{M}\left(x_{1}, x^{\prime}\right) d x_{1} .
$$

So, if we integrate over $x^{\prime} \in \mathbb{R}^{n-1}$, we obtain that

$$
\int_{r+S} h_{M}(x) d x=\int_{S} h_{M}(x) d x=\kappa_{M}
$$

Now, given any $y \in \mathbb{R}^{n}$, we notice that $-y+S=-y_{1}+S$, and thus

$$
\int_{S} h_{M}(x-y) d x=\int_{-y+S} h_{M}(x) d x=\kappa_{M} .
$$

Moreover, fixed $x_{1} \in \mathbb{R}$, we have that the map $\mathbb{R}^{n-1} \ni x^{\prime} \mapsto K\left(x_{1}, x^{\prime}\right)$ is radially symmetric and decreasing, therefore $K^{x_{1}, *}\left(x^{\prime}\right)=K\left(x_{1}, x^{\prime}\right)$. Accordingly, $h_{M}^{x_{1}, *}\left(x^{\prime}\right)=h_{M}\left(x_{1}, x^{\prime}\right)$. Also, for any fixed $x_{1} \in \mathbb{R}$, we have that $\chi_{F^{x_{1}}}^{*}=\chi_{\left(F^{\left.x_{1}\right)^{*}}\right.}=\chi_{F^{x_{1}}, *}$. Thus, fixed $x_{1} \in \mathbb{R}$, we use the Riesz rearrangement inequality (see e.g. Theorem 3.7 in [10]) and we obtain

$$
\begin{aligned}
& \int_{\mathbb{R}^{n-1}} \int_{\mathbb{R}^{n-1}} \chi_{F}\left(x_{1}, x^{\prime}\right) h_{M}\left(x_{1}-y_{1}, x^{\prime}-y^{\prime}\right) \chi_{F}\left(y_{1}, y^{\prime}\right) d x^{\prime} d y^{\prime} \\
= & \int_{\mathbb{R}^{n-1}} \int_{\mathbb{R}^{n-1}} \chi_{F^{x_{1}}}\left(x^{\prime}\right) h_{M}\left(x_{1}-y_{1}, x^{\prime}-y^{\prime}\right) \chi_{F^{y_{1}}}\left(y^{\prime}\right) d x^{\prime} d y^{\prime} \\
\leqslant & \int_{\mathbb{R}^{n-1}} \int_{\mathbb{R}^{n-1}} \chi_{F^{x_{1}}}^{*}\left(x^{\prime}\right) h_{M}^{*}\left(x_{1}-y_{1}, x^{\prime}-y^{\prime}\right) \chi_{F^{y_{1}}}^{*}\left(y^{\prime}\right) d x^{\prime} d y^{\prime} \\
= & \int_{\mathbb{R}^{n-1}} \int_{\mathbb{R}^{n-1}} \chi_{F^{x_{1}}, *}\left(x^{\prime}\right) h_{M}\left(x_{1}-y_{1}, x^{\prime}-y^{\prime}\right) \chi_{F^{y_{1}}, *}\left(y^{\prime}\right) d x^{\prime} d y^{\prime} \\
= & \int_{\mathbb{R}^{n-1}} \int_{\mathbb{R}^{n-1}} \chi_{F^{\star}}\left(x_{1}, x^{\prime}\right) h_{M}\left(x_{1}-y_{1}, x^{\prime}-y^{\prime}\right) \chi_{F^{\star}}\left(y_{1}, y^{\prime}\right) d x^{\prime} d y^{\prime} .
\end{aligned}
$$

Now we integrate over $x_{1} \in[-1 / 2,1 / 2]$ and $y_{1} \in[-1 / 2,1 / 2]$ and we obtain that

$$
\int_{S} \int_{S} \chi_{F}(x) h_{M}(x-y) \chi_{F}(y) d x d y \leqslant \int_{S} \int_{S} \chi_{F^{\star}}(x) h_{M}(x-y) \chi_{F^{\star}}(y) d x d y .
$$

On the other hand, if $x \in S$, we have that $\chi_{F}(x)=1-\chi_{S \backslash F}(x)$, therefore

$$
\begin{aligned}
& \int_{S} \int_{S} \chi_{F}(x) h_{M}(x-y) \chi_{F}(y) d x d y \\
= & \int_{S} \int_{S} h_{M}(x-y) \chi_{F}(y) d x d y-\int_{S} \int_{S} \chi_{S \backslash F}(x) h_{M}(x-y) \chi_{F}(y) d x d y \\
= & \kappa_{M}|F|-\int_{S \backslash F} \int_{F} h_{M}(x-y) d x d y
\end{aligned}
$$

thanks to (B.1). Similarly, we have that

$$
\int_{S} \int_{S} \chi_{F^{\star}}(x) h_{M}(x-y) \chi_{F^{\star}}(y) d x d y=\kappa_{M}\left|F^{\star}\right|-\int_{S \backslash F^{\star}} \int_{F^{\star}} h_{M}(x-y) d x d y .
$$

Therefore, recalling (B.2), we obtain that

$$
\kappa_{M}|F|-\int_{S \backslash F} \int_{F} h_{M}(x-y) d x d y \leqslant \kappa_{M}\left|F^{\star}\right|-\int_{S \backslash F^{\star}} \int_{F^{\star}} h_{M}(x-y) d x d y .
$$


Hence, using that $\left|F^{\star}\right|=|F|$ and that $h_{M} \leqslant K$, we obtain that

$$
\int_{S \backslash F^{\star}} \int_{F^{\star}} h_{M}(x-y) d x d y \leqslant \int_{S \backslash F} \int_{F} K(x-y) d x d y .
$$

Now we observe that, by Fatou Lemma,

$$
\liminf _{M \rightarrow+\infty} \int_{S \backslash F^{\star}} \int_{F^{\star}} h_{M}(x-y) d x d y \geqslant \int_{S \backslash F^{\star}} \int_{F^{\star}} K(x-y) d x d y,
$$

thus we can pass to the limit (B.3) and obtain the desired result.

In the light of Proposition 13, we have that the cylindrical symmetry assumption for the set of competitors in $\mathscr{K}$ (recall the definition on page 2) can be weakened. Indeed, it is not necessary to suppose that the competitors are a priori cylindrically symmetric, since the cylindrical rearrangement makes the energy functional decrease. It would be interesting to weaken also the assumption that the set is a priori decreasing with respect to $x_{1} \in[0,1 / 2]$. In principle, a periodic version of the cylindrical rearrangement should prove that the energy also decreases under monotone rearrangement in the $x_{1}$ variable. Though this property is in accordance with the intuition and with some numerical simulations, it is not immediate to give a rigorous proof of it, due to the presence of competing terms in the sum that defines the functional, so we leave this as an open problem.

\section{REFERENCES}

[1] N. Abatangelo and E. Valdinoci, A Notion of Nonlocal Curvature, Numer. Funct. Anal. Optim. 35 (2014), no. 7, 793-815.

[2] L. Ambrosio, N. Fusco and D. Pallara, Functions of bounded variation and free discontinuity problems. Oxford Mathematical Monographs. The Clarendon Press, Oxford University Press, New York, 2000.

[3] X. Cabré, M. M. Fall, J. Solà-Morales, T. Weth, Curves and surfaces with constant nonlocal mean curvature: meeting Alexandrov and Delaunay, http://arxiv.org/abs/1503.00469

[4] L. Caffarelli, J.-M. Roquejoffre and O. Savin, Nonlocal minimal surfaces, Comm. Pure Appl. Math. 63 (2010), no. 9, 1111-1144.

[5] L. Caffarelli and E. Valdinoci, Uniform estimates and limiting arguments for nonlocal minimal surfaces, Calc. Var. Partial Differ. Equ. 41 (2011), no. 1-2, 203-240.

[6] J. Dávila, M. del Pino and J. Wei, Nonlocal Minimal Lawson Cones, preprint, http://arxiv.org/abs/1303.0593

[7] C. Delaunay, Sur la surface de révolution dont la courbure moyenne est constante, J. Math. Pures Appl. 6 (1841), 309-320.

[8] A. Di Castro, M. Novaga, B. Ruffini and E. Valdinoci, Nonlocal quantitative isoperimetric inequalities, to appear in Calc. Var. Partial Differential Equations, DOI 10.1007/s00526-015-0870-x.

[9] A. Figalli, N. Fusco, F. Maggi, V. Millot and M. Morini, Isoperimetry and stability properties of balls with respect to nonlocal energies, Commun. Math. Phys. 336 (2015), no. 1, 441-507.

[10] E. H. Lieb, M. Loss, Analysis. Second edition. Graduate Studies in Mathematics, 14. American Mathematical Society, Providence, RI, 2001.

(Juan Dávila and Manuel del Pino) Departamento de Ingeniería Matemática and Centro de Modelamiento Matemático, Universidad de Chile, Casilla 170 Correo 3, 8370459 Santiago, Chile

E-mail address: jdavila@dim.uchile.cl, delpino@dim.uchile.cl

(Serena Dipierro) Institut für Analysis und Numerik, Otto-von-Guericke-Universität Magdeburg, UniVersitätsplatz 2, 39106 Magdeburg, Germany

E-mail address: serena.dipierro@ed.ac.uk

(Enrico Valdinoci) Weierstrass Institut für Angewandte Analysis und Stochastik, Hausvogteiplatz 11A, 10117 Berlin, Germany

E-mail address: enrico.valdinoci@wias-berlin.de 